\title{
Towards a Unified Description of Light Ion Fusion Cross Section Excitation Functions
}

K. W. Zimmer

Editor: H. Rebel Institut für Kernphysik 

KERNFORSCHUNGSZENTRUM KARLSRUHE

Institut für Kernphysik

KfK 5386

\title{
TOWARDS A UNIFIED DESCRIPTION OF LIGHT ION FUSION CROSS SECTION EXCITATION FUNCTIONS
}

\author{
K. W. Zimmer*
}

Editor: H. Rebel

* Invited scientist from Institute of Atomic Physics, IPNE, Bucharest, Romania.

Deceased in March, 16, 1994

Kernforschungszentrum Karlsruhe GmbH, Karlsruhe 
Als Manuskript gedruckt

Für diesen Bericht behalten wir uns alle Rechte vor

Kernforschungszentrum Karlsruhe $\mathrm{GmbH}$ Postfach 3640, 76021 Karlsruhe

ISSN 0303-4003 


\begin{abstract}
A description of light heavy-ion fusion, taking into account both entrancechannel characteristics and compound-nucleus properties, is derived within a unified theory of nuclear reactions. The dependence of the imaginary fusion potential on the level density of the compound nucleus is revealed. The $12 \mathrm{C}+12 \mathrm{C}$, $12 \mathrm{C}+14 \mathrm{~N}, 10 \mathrm{~B}+16 \mathrm{O}$ and $16 \mathrm{O}+16 \mathrm{O}$ fusion cross sections are calculated for $\mathrm{E}_{\mathrm{cm}} \leqq 120 \mathrm{MeV}$ and compared with experimental data. The excitation energy dependence of the level-density parameter of $24 \mathrm{Mg}, 26 \mathrm{Al}$ and $32 \mathrm{~S}$ is inferred below $5 \mathrm{MeV} / \mathrm{A}$. A realistic nuclear level-density model, describing the experimental level-density parameters of highly excited nuclei, is shown to be consistent with both the global features and details of the fusion cross section. $12 \mathrm{C}+12 \mathrm{C}$ and $16 \mathrm{O}+16 \mathrm{O}$ fusion cross section oscillations are predicted at large excitation energies, reflecting the structure of the level density of the highly excited light compound nuclei. Differences of the $12 \mathrm{C}+14 \mathrm{~N}$ and $10 \mathrm{~B}+16 \mathrm{O}$ fusion reaction mechanisms are discussed in terms of specific entrance-channel characteristics.
\end{abstract}




\section{HIN $Z U$ EINER EINHEITLICHEN BESCHREIBUNG DER ANREGUNGSFUNK'TIONEN FÜR DIE FUSION LEICHTER IONEN}

Im Rahmen der einheitlichen Theorie der Kernreaktionen wird eine Beschreibung der Fusion leichter Schwerionen unter Berücksichtigung der Eingangskanal-Charakteristika und der Compound-Kern-Eigenschaften gegeben. Die Abhängigkeit des imaginären Fusionspotentials von der Niveaudichte des Compound-Kernes wird deutlich gemacht. Die $12 \mathrm{C}+12 \mathrm{C}$, $12 \mathrm{C}+14 \mathrm{~N}, 10 \mathrm{~B}+160$ und $16 \mathrm{O}+160$ Fusionswirkungsquerschnitte werden für den Energiebereich $\mathrm{E}_{\mathrm{cm}} \leqq 120 \mathrm{MeV}$ berechnet und mit experimentellen Daten verglichen. Die Energieabhängigkeit der Niveaudichte-Parameter von $24 \mathrm{Mg}$, $26 \mathrm{Al}$ und $32 \mathrm{~S}$ wird für Anregungsenergien unterhalb $5 \mathrm{MeV} / \mathrm{A}$ abgeleitet. Die Konsistenz eines realistischen Niveaudichte-Modells mit den globalen Eigenschaften und Details der Fusionsquerschnitte wird aufgezeigt. Für $12 \mathrm{C}+12 \mathrm{C}$ und $16 \mathrm{O}+16 \mathrm{O}$ wird bei hohen Anregungsenergien ein oszillatorisches Verhalten der Fusion-Anregungsfunktionen vorausgesagt, das die Niveaudichte der hochangeregten Compound-Kerne widerspiegelt. Unterschiede in den $12 \mathrm{C}+14 \mathrm{~N}$ und $10 \mathrm{~B}+16 \mathrm{O}$ Fusionsmechanismen werden als Folge spezifischer Eingangskanal-Charakteristika diskutiert. 


\section{Content}

\section{Page}

1. Introduction 1

2. Optical Model Description of Fusion 4

3. The Nuclear Level-density Model 10

4. Fusion Excitation Functions 21

$\begin{array}{lll}\text { 5. Conclusions } & 27\end{array}$

$\begin{array}{ll}\text { References } & 30\end{array}$ 
"Ein stiller Geist ist jahrelang geschäftig.

Die Zeit nur macht die feine Gärung kräftig."

Johann Wolfgang von Goethe (Faust 1)

\section{Introduction}

Since the first studies of the limiting conditions of nuclear fusion $[1,2]$, the identification of specific entrance-channel [3-5] and compound nucleus [6-10] characteristics, which determine the salient features of the fusion cross sections in various energy regimes, has been a topic of considerable interest. Ignoring usually the spins of the colliding nuclei, the sharp cut-off approximation has been invoked. It assumes that relative angular momenta $\ell$ smaller than a particular critical angular momentum $J_{c r}$ contribute to complete fusion, while higher values of $\ell$ are associated with direct (peripheral) processes. At lower incident energies (denoted region I) the fusion cross section of exhausts nearly the total reaction cross section $\sigma_{\mathrm{R}}$ and the critical value $J_{c r}$ is essentially determined by the entrance-channel properties. However, especially for light and symmetric mass systems limitations for compound nucleus formation are also evident at higher excitation energies (denoted regions $I I$ and III) where $\sigma_{F}$ is significantly smaller than $\sigma_{R}$. For different entrance channels, leading to the same compound nucleus, a single locus of $J_{c r}$ develops in the angular momentum-excitation energy $U$ plane, to the left of the Yrast line $[6,11,12]$. The statistical Yrast line, defined by shifting the Yrast line by an amount $\Delta Q$ parallel to the energy axis, describes this locus well $[7,10]$. From point of view of the statistical approach to level densities [13], the statistical Yrast line represents the locus of a constant compound nucleus state density $\omega(\mathrm{U}, \mathrm{M})[14,15]$ :

$$
\omega\left(U, M=J_{c r}(U)\right)=\rho(\Delta Q)=\text { constant }
$$

Here $\rho$ is the level density and $M$ the total angular momentum projection. At higher energies (region III) experimental data indicate a saturation of $J_{c r}$ for 
light systems $[11,12,16-18]$ near the value where the fission barrier vanishes according to the rotating liquid drop model $[19,20]$.

There is some theoretical evidence [21] that the level density of highly excited nuclei is drastically reduced as compared to the predictions of the traditional description [13], mainly as a consequence of the finite depth of the self-consistent potential which limits the relevant single particle space to a finite number of states. Because of the induced distortion of the contour lines of $\rho(U, J)[22]$ and $\omega$ $(U, M)$ towards smaller $J, M$ at higher excitation energies, an alternative explanation of the compound nucleus limitations to fusion in region III might be possible. Infact, for $A=114-160$ nuclei a considerable decrease of the leveldensity parameter, varying from $a=\mathrm{A} / 8\left[\mathrm{MeV}^{-1}\right]$ at low $\varepsilon=\mathrm{U} / \mathrm{A}$ to $a=\mathrm{A} / 13[\mathrm{MeV}$ 1) at $\varepsilon=3 \mathrm{MeV} / \mathrm{A}$ has been observed [26-28]. For light nuclei ( $\mathrm{A} \leqq 40$ ), a value $a=\mathrm{A} / 8\left[\mathrm{MeV}^{-1}\right]$ has been inferred with a similar tendency at higher excitation energies $\varepsilon=2-3 \mathrm{MeV} / \mathrm{A}[29]$.

For several light systems, especially for the spin-zero cases $12 \mathrm{C}+12 \mathrm{C}[30,31,34]$, $16 \mathrm{O}+160[32-34]$ and the $12 \mathrm{C}+160[34,35]$, an oscillation pattern is superimposed on the general trend of $\sigma_{\mathrm{F}}$ below $\mathrm{E}_{\mathrm{cm}} \approx 30 \mathrm{MeV}$. The structures have been related to entrance channel characteristics $[36,37]$. But these as well as the smooth $12 \mathrm{C}+16 \mathrm{~N}, 10 \mathrm{~B}+160$ fission excitation functions have been equally well described by a phenomenological parametrization of the fusion transmission coefficients $\mathrm{T}_{\ell}^{\mathrm{F}}$, which encompasses the constraints due to the effective interaction in the entrance channel at low energy and to the angular momentum dependent compound nuclear level density at higher energy [8,9]. For light nuclei $(\mathrm{A} \leqq 40)$ combinatorial calculations [15] reveal structures in $\omega(U, M), \rho(U$, $J$ ) even at high $U$. A significant correlation (eq. 1.1) between $J_{c r}(U)$ of the $12 \mathrm{C}+12 \mathrm{C}, 16 \mathrm{O}+16 \mathrm{O}$ and $16 \mathrm{O}+24 \mathrm{Mg}$ systems with oscillating $\sigma_{\mathrm{F}}$ and the structured contours of the compound nucleus state density $\omega(U, M)[14,15]$ has been observed. For $\mathrm{E}_{\mathrm{cm}} \geqq 30 \mathrm{MeV} \sigma_{\mathrm{F}}$ has been measured in relatively large 
energy steps, leaving the question open if corresponding structures exists at higher energy. Extrapolating eq. (1.1) to higher energies suggests that oscillations of $\sigma_{F}$ may persist. It is important to understand which entrance channel characteristics allow $\sigma_{\mathrm{F}}$ to be sensitive or insensitive to such details of compound-nucleus level density.

Actually, the unified description of excitation functions of light systems covering a broad energy range requires the proper consideration of the competition between direct processes and fusion for the flux absorbed from the entrance channel, in terms of ingredients from both nuclear dynamics and structure. In this sense, it has been shown phenomenologically $[8,9,39-41]$ and justified in the frame-work of general reaction theories $[15,25,42-45]$ that the optical model description of fusion is a simple but relevant and efficient procedure. In analogy with the expression of $\sigma_{\mathrm{R}}[46]$ (and $\mathrm{T}_{\ell}$ ) $\sigma_{\mathrm{F}}$ and $\mathrm{T}_{\ell}^{\mathrm{F}}$ are expressed [39] in terms of optical model wave functions and $W_{F}$, the imaginary part of the potential which is responsible for absorption due to fusion. Formal expressions of the optical potential $U=V+i W$ and of $W_{F}$, derived in the frame-work of projection operator $[44,45]$ formalisms, include the effects of channel-coupling on fusion cross sections (polarization effects in $V$, contributions to $W_{F}$ related to particular fusion mechanisms) and apply to the case of heavy assymmetric mass systems at energies below and around the Coulomb barrier. For light systems, however, the distinct mark of properties of the highly excited compound nucleus $J_{c r}$ on of has a correspondent in $W_{F}[15]$, when isolated in the formal expression of the optical model potential within the unified theory of nuclear reactions [47-49].

In this paper we investigate the relation between the features of light heavy-ion fusion and specific properties of the level density of highly excited light nuclei. For reference the formalism is recalled in sect. 2 and approximations of the optical model potential, $W_{F}$ and $\mathrm{T}^{\mathrm{F}}{ }_{\ell}$ are derived and their dependence on quantities describing the reaction dynamics and of the compound nucleus level 
density is discussed. On this basis a new method to study the excitation energy dependence of the level-density parameter of highly excited light nuclei using fusion excitation functions is proposed and illustrated for $24 \mathrm{Mg}, 26 \mathrm{Al}$ and $32 \mathrm{~S}$. A realistic level-density model is presented and its predictions are compared with the experimental information on the level density of highly excited nuclei. Specific properties of the level density of highly excited light nuclei, e.g. the parity dependence, are discussed in the frame of this model. Exact combinatorial calculations of $J$, parity (II), isopin (T) dependent level densities of $24 \mathrm{Mg}, 26 \mathrm{Al}$ and $32 \mathrm{~S}$ are performed for $\varepsilon \leqq 5 \mathrm{MeV} / \mathrm{A}$. The results are used for the unified description of $12 \mathrm{C}+12 \mathrm{C}, 12 \mathrm{C}+14 \mathrm{~N}, 10 \mathrm{~B}+16 \mathrm{O}$ and $16 \mathrm{O}+16 \mathrm{O}$ total fusion data. Oscillations related to the structures in the compound nucleus level density are predicted in the $12 \mathrm{C}+12 \mathrm{C}$ and $16 \mathrm{O}+16 \mathrm{O}$ fusion excitation functions at high energy. The decrease of the sensitivity of $\sigma_{F}$ to the structures in the compound nucleus level density with increasing channel spin is illustrated. Differences in the $12 \mathrm{C}+14 \mathrm{~N}$ and $10 \mathrm{~B}+16 \mathrm{O}$ fusion mechanisms are discussed. It is shown that the anomalies of the $10 \mathrm{~B}+16 \mathrm{O}$ fusion cross section at moderate energies are consistent with a significant contribution of non-elastic fusion.

\section{Optical Model Description of Fusion}

Following [47-49], the operators P, I, N, Q are defined to project on to the open channel, entrance channel, non-elastic channel and closed channel subspaces, respectively. Let $\mathbf{H}$ be the total Hamiltonian of the system. The projectives $\mathrm{O}_{\mathrm{KL}}=\mathrm{KOL}(\mathrm{K}, \mathrm{L}=\mathrm{P}, \mathrm{I}, \mathrm{N}, \mathrm{Q})$ are defined for an operator $\mathrm{O}$. The strongly energy dependent compound nucleus processes are related to the finite number of eigenstates $\mid \mathrm{x}_{\mathrm{S}}>$ of $\mathrm{H}_{\mathbf{Q Q}}$ which lie in the interval $\Delta \mathrm{E}$ around $\mathrm{E}$. We define the projection operator $\mathbf{R}=\Sigma\left|\mathbf{x}_{\mathrm{S}}><\mathrm{x}_{\mathrm{S}}\right|$ on the resonance subspace. The true resonance states $\left|X_{t}\right\rangle$ are solutions of the eigenvalue problem $\left(E_{t}-H_{R R}-W_{R R}\right) \mid X_{t}>=0$, where $\left.W_{R R}=H_{R P} /\left(E^{+}-H^{\prime}\right)\right) H_{P R}$ 
and $H^{\prime}=H_{P P}+H_{P Q}((\mathbf{Q}-\mathbf{R})) /\left(E-H_{Q Q}\right) H_{Q P}$.

The detailed formal expression of the entrance-channel optical potential [47-49] reads as

$\mathrm{U}=\mathrm{V}_{\mathrm{II}}+\mathrm{H}_{\mathrm{IR}} \mathrm{YH}_{\mathrm{RI}}+\left(\mathrm{H}^{\prime}{ }_{\mathrm{IN}}+\mathrm{H}_{\mathrm{IR}} \mathrm{YH}_{\mathrm{RN}}\right) \mathrm{G}_{\mathrm{NN}}\left(\mathrm{H}_{\mathrm{NR}} \mathrm{YH}_{\mathrm{RI}}+\mathrm{H}^{\prime}{ }_{\mathrm{NI}}^{\prime}\right.$

where

$\mathrm{V}_{\mathrm{II}}=\mathrm{H}_{\mathrm{II}}-\mathrm{T} \quad$ ( $\mathrm{T}$ is the kinetic energy),

$\mathbf{Y}=\mathbf{Z} /\left(1+\mathbf{Z} \mathbf{W}_{\mathbf{R R}}\right), \mathbf{Z}=-i_{\mathbf{I}} \mathbf{R} / \Delta E$

and finally

$\mathrm{G}_{\mathrm{NN}}=1 /\left(E^{+}-\mathrm{H}^{\prime} \mathrm{NN}^{-\mathrm{H}_{\mathrm{NR}}} \mathrm{YH}_{\mathrm{RN}}\right)$

is the Green's operator.

Due to the fact that the formal manipulations leading to (2.1) preserve rotational invariance, parity and other symmetries of $\mathbf{H}$, the operators arising in the expression of $\mathbf{U}$ and $U$ itself act within or couple subspaces with the same $J, M, \Pi$, etc. These dynamical angular-momentum coupling effects on elastic scattering (and on absorption from the entrance-channel) are reglected and any vector coupling, in the case of non-zero projectile and target spins $I_{1}, I_{2}$ is implied. The nonlocal radial dependence of $U$ is formally obtained as the partial expectation value $U_{J l s}=(J l s \Pi|U| J l s \Pi)$ where $\left.\mid J l s \Pi\right)$ denotes the wave function of the intrinsic state of projectile, target, and the relative angular motion $e$ in the entrance-channel coupled with the entrance-channel spin $s$ to $J$.

The operators $\mathrm{H}_{K R} \mathrm{YH}_{\mathrm{RL}}(\mathrm{K}, \mathrm{L}=\mathrm{I}, \mathrm{N})$ describe effects of transitions mediated by compound-nucleus states, where $\mathrm{Y}$ accounts for effects due to the finite life-time and to the correlation of compound-nucleus states, arising from their coupling to the $P$ space. If $\Delta \mathrm{E}$ is large in comparison with the effective coupling $\mathbf{W}_{\mathbf{R} R}$, one may replace $\mathrm{Y}$ by $\mathrm{Z}$, leading to the approximation

$$
\begin{aligned}
& \mathbf{H}_{\mathbf{K} \mathbf{R}} \mathbf{Y} \mathbf{H}_{\mathbf{R L}} \simeq i \operatorname{in\rho }(U, J, \Pi) \\
& {\left[\mathbf{H}_{\mathbf{K} \mathbf{R}} \mid(X)\left(X \mid \mathbf{H}_{\mathbf{R} \mathbf{L}}\right]=-i \rho(U, J, \Pi)\left[\mathbf{H}_{\mathbf{K} \mathbf{R}} \mid x\right)\left(x \mid \mathbf{H}_{\mathbf{R L}}\right]\right.}
\end{aligned}
$$


where the brackets [ ] signify averaging over the relevant compound-nucleus states. The short equilibration time of about $2.10^{-22} \mathrm{~s}$ in light ion collisions [50] and the empirical systematics of compound-nucleus widths [51] indicate the scaling law $\Delta \mathrm{E} \sim \sqrt{ } \varepsilon$ so that $\Delta \mathrm{E}$ can be chosen to be a few $\mathrm{MeV}$ (when the excitation energy $\varepsilon \leqq 5 \mathrm{MeV} / \mathrm{A}$ ).

A transparent expression of $U$ results

$$
\begin{aligned}
& U_{J l s \Pi}=\left(J l s \Pi \mid\left\{\mathbf{V}_{\mathbf{I I}}-\operatorname{irp}\left[\mathbf{H}_{\mathbf{I R}} \mid x\right)\left(x \left[\mathbf{H}_{\mathbf{R I}} \mid+\left(\mathbf{H}^{\prime}{ }_{\mathbf{I N}}-i \operatorname{in\rho }\left[\mathbf{H}^{\prime}{ }_{\mathbf{I R}} \mid x\right)\left(x \mid \mathbf{H}_{\mathbf{R N}}\right]\right)\right.\right. \text { * }\right.\right. \\
& \left.\left(E^{+}-\mathbf{H}^{\prime}{ }_{\mathbf{N N}}-i \operatorname{in\rho }\left[\mathbf{H}_{\mathbf{N R}} \mid x\right)\left(x\left[\mathbf{H}_{\mathbf{R N}}\right]\right)^{-1}\left(\mathbf{H}^{\prime}{ }_{\mathbf{N I}}-i \operatorname{in\rho }\left[\mathbf{H}_{\mathbf{N R}} \mid x\right)\left(x \mid \mathbf{H}_{\mathbf{R I}}\right]\right)\right\} \mid J l s \Pi\right) \text {. }
\end{aligned}
$$

$\mathbf{H}^{\prime}{ }^{\prime} N$ does not commute with $\left[H_{N R} \mid x\right)\left(x \mid H_{R N}\right]$, reflecting the multistep nature of the effects contained in the optical potential. Generally, $\left[H_{K R} \mid x\right)\left(x \mid H_{R L}\right]$ with $(K$, $\mathbf{L}=\mathbf{I}, \mathbf{N}$ ) is expected to be small due to the complicated nature of the compoundnucleus.

Also in region $\amalg$ and $\amalg$ (for $J \geqq J_{c r}$ ) the density of compound-nucleus levels with specified $J, \Pi, T=0$ is lower than $50-100$ levels $/ \mathrm{MeV}$. Therefore compoundnucleus effects in (2.2) are treated only up to the first order. For simplicity also the non-local expressions $\left[\left(J / s \Pi\left|O_{I R}\right| x\right)\left(x\left|O_{R I}\right| J l s \Pi\right)\right]$ are replaced with the local ones $\left[\left|\left(J l s \Pi\left|O_{R I}\right| x\right)\right|^{2}\right]$. Then the following approximations for $V$ and $W$ are obtained

$$
\begin{aligned}
& V_{J l s \Pi}=\left(J l s \Gamma|| \mathrm{V}_{\mathrm{II}}+\mathbf{H}^{\prime}{ }_{\mathrm{IN}} \mathrm{P}\left(\left(E^{\prime}-\mathrm{H}^{\prime}{ }_{\mathrm{NN}}\right)^{-1}\right) \mathrm{H}^{\prime}{ }_{\mathrm{NI}} \mid J l s \Pi\right)-\mathrm{r}^{2} \boldsymbol{\rho}(U, J, \Pi) E_{J l s \Pi} \\
& W_{J l s \Pi}=W_{d l s \Pi}^{D}+W^{F} l_{s \Pi \Pi} \\
& W_{d l s \Pi}^{D}=-\mathrm{nl}\left(J l s \Pi\left|\mathrm{H}_{1 \mathrm{~N}}{ }_{1 \mathrm{~N}} \delta\left(E-\mathrm{H}^{\prime}{ }_{\mathrm{NN}}\right) \mathrm{H}^{\prime}{ }_{\mathrm{NI}}\right| J l s \mathrm{I}\right) \leq 0 \\
& W_{J l s \Pi}^{F}=-\mathrm{II} \rho(U, J, \mathrm{II})\left(A_{J l s \mathrm{II}}+B_{J l s I I}+C_{J l s I I}+D_{J l s I I}\right) \leq 0 \\
& A_{J l s \Pi}=\left[\left|\left(J l s \Pi|| \mathbf{H}_{\mathbf{I R}} \mid x\right)\right|^{2} \mathrm{I} \geq 0\right. \\
& B_{J l s \Pi}=\mathbf{n}^{2}\left[\left|\left(J l s \Pi\left|\mathbf{H}_{\mathbf{I N}} \delta\left(E-\mathbf{H}^{\prime}{ }_{N N}\right) \mathbf{H}_{\mathrm{NR}}\right| x\right)\right|^{2}\right] \geq 0 \\
& C_{J l s I}=\left[\left|\left(J l s \Pi\left|\mathbf{H}^{\prime}{ }_{\mathrm{IN}} \mathrm{P}\left(\left(E-\mathbf{H}^{\prime}{ }_{\mathrm{NN}}\right)^{-1}\right) \mathbf{H}_{\mathrm{NR}}\right| x\right)\right|^{2}\right] \geq 0
\end{aligned}
$$




$$
\begin{aligned}
& \left.D_{J l s \Pi}=2 R e\left\{l \mid\left(J l s \Pi\left|\mathbf{H}^{\prime}{ }_{I N} \mathrm{P}\left(\left(E-\mathbf{H}^{\prime}{ }_{N N}\right)^{-1}\right) \mathbf{H}_{\mathrm{NR}}\right| x\right)\left(x\left|\mathbf{H}_{\mathbf{R I}}\right| J l s \Pi\right)\right]\right\} \simeq 0 \\
& E_{J l s \Pi}=2 R e\left\{\left[\left(J l s \Pi\left|\mathbf{H}^{\prime}{ }_{\mathrm{IN}} \delta\left(E-\mathbf{H}^{\prime}{ }_{\mathrm{NN}}\right) \mathbf{H}_{\mathrm{NR}}\right| x\right)\left(x\left|\mathbf{H}_{\mathbf{R I}}\right| J l s \Pi\right)\right]\right\} \simeq 0
\end{aligned}
$$

Neglecting of $D$ and $E$ on random-phase arguments, $V$ is determined by direct contributions and $W$ is the sum of the direct term $W^{D}$ and the fusion term $W F$, both to be defined negative. The part $W D$ is related to the polarization term in $V$ by a dispersion relation and WF displays the strong $U, J$ dependence of the compound-nucleus level density. The terms $A, B$ and $C$ describe different mechanisms: (A) fusion in the entrance-channel, (B) fusion while propagating onenergy-shell in the $\mathbf{N}$ subspace after a direct transition from the entrancechannel, (C) fusion in non-elastic channels virtually populated from the entrance-channel by direct couplings.

For $J \leqq J_{\text {cr, }}$, the terms $\left[\mathbf{H}_{\mathbf{K R}} \mid \mathbf{x}\right)\left(\mathrm{x} \mid \mathbf{H}_{\mathbf{R L}}\right](\mathbf{K} \neq \mathbf{L}=\mathbf{I}, \mathbf{N})$, although small, are multiplied with the strongly increasing $\rho(U, J)$. Neglecting the $\operatorname{direct} \mathbf{I}, \mathbf{N}$ channel couplings in the third term of (2.2), $\mathrm{W}_{\mathrm{Jl}}$ II contains only terms related to initial compound-nucleus formation from the entrance-channel, therefore $\mathrm{W}_{\mathrm{Jls} \Pi} \simeq \mathrm{WF}_{\mathrm{JlsII}}$.

The transition between the extremes of low $\rho$ for $J>J_{c r}$ and high $\rho$ for $J<J_{c r}$ is assumed to occur sufficiently fast. For J values above the Yrast values $\rho(U, J)=0$ and the optical potential is influenced by direct effects only. The transmission coefficients for fusion are expressed as

$$
T^{F}{ }_{J l \mathrm{II}}=8 \mathrm{r} k / \hbar^{2} \int_{o}^{\infty} d r\left|\Psi_{J l s \mathrm{II}}\right|^{2}\left(-W_{J l s \Pi}^{F}\right)
$$

and satisfy the inequality $0 \leqq \mathrm{~T}^{\mathrm{F}} \mathrm{Jl}_{\mathrm{s} \Pi} \leqq \mathrm{T}_{\mathrm{Jl}} \Pi \leqq 1$. Here $\Psi_{\mathrm{Jls} \Pi}$. is the radial wave function generated with the optical potential $U_{J_{1 s} l 1}$. The fusion cross section is now written in analogy with the reaction cross section as 


$$
\sigma_{F}=\mathrm{n} / k^{2} \sum_{l=0} \sum_{s=\left|I_{1}-I_{2}\right|}^{I_{1}+I_{2}}\left[1+n\left((-1)^{(l+s)}\right] \sum_{J=|l-s|}^{l+s} g_{J} T^{F^{\prime}}{ }_{J l s \mathrm{II}}\right.
$$

where $\mathrm{k}$ is the the wave vector, $\mathrm{n}$ is 1 for identical ions and 0 otherwise, $g_{J}=(2 J+1) /\left(2 I_{1}+1\right) /\left(2 I_{2}+1\right)$.

The calculations performed in this paper use phenomenological Woods-Saxon optical potentials $V+i W$ with energy dependent parameters taken from Refs. [52], [53], [11], [54], and describing elastic scattering and reaction cross section. A more detailed analysis of the radial, energy, angular momentum dependence of the contributions $A, B, C$ is left for a later work. Here, the influence of these dynamical factors is treated on the simplifying assumption that the energy and $J$ dependence of $W F$ is dominated by $\rho$. Usually, the radial dependence of $F=A+B+C$ is assumed to depend on the overlap of two spheres with radii $R_{i}=r_{F} A_{i} 1 / 3$ and $r_{F}=1.35 \mathrm{fm}$ i.e. on the separation of the two centers, roughly simulating the volume-type radial dependence of $A$. Additional surface type contributions of $B, C$ are assumed to be absorbed by increasing the value of $r_{0}$. The magnitude of $F$ is normalized by the condition

$$
F\left(r=0, E_{c m}\right)=\mathrm{k}\left|W\left(r=0, E_{c m}\right)\right|
$$

The constant $\mathrm{K}$ is considered to be equal for all systems: $\mathrm{K}=510^{-3}$ when isospin is considered a conserved quantum number, and $\mathrm{k}=2.510^{-3}$ otherwise. This makes $\mathrm{W}^{\mathrm{F}} \mathrm{Jls} \Pi(0) \simeq \mathrm{W}(0)$ for $\rho(U, J, \Pi, \mathrm{T}=0) \simeq 64$ levels $/ \mathrm{MeV}$ and $\rho(U, J, \Pi) \simeq 127$ levels/MeV, respectively.

The analysis of this paper attempts to explore the sensitivity of fusion cross sections to details of the compound nucleus level density $\rho(U, J, \Pi)$ at higher excitation energies. Experimental $12 \mathrm{C}+12 \mathrm{C}[16,31,34], 12 \mathrm{C}+14 \mathrm{~N}[11,17,31,34]$ and $160+160$ [56] fusion cross sections and results of quantum molecular dynamics calculations [57] are compared with the fusion excitation functions calculated in the framework of the present model. The familiar expression [13] 


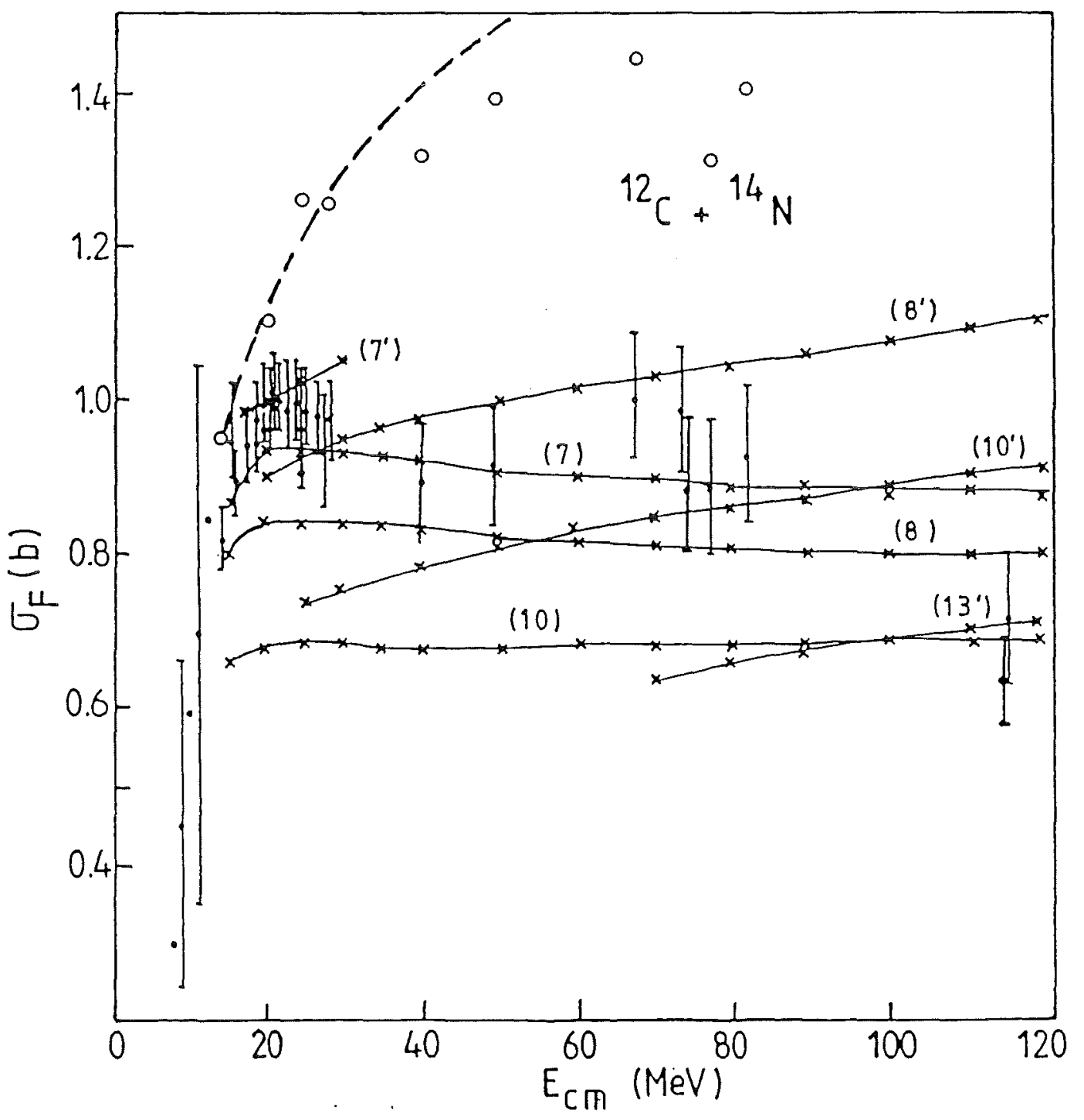

Fig. 1: Total fusion cross sections for $12 C+14 N$ : comparison of values calculated within the present model (solid line with crosses) for different values of the inverse level-density parameter Ala attached to the curves in paranthesis using either a constant value of the moment of inertia $\theta$ or an experimentally suggested spin cut off factor o (value of Ala primed). Experimental values $\sigma_{F}$ are taken from refs. $11,17,31,34,55$. The open circles represent the total reaction cross section [17], compared with optical model predictions (dashed). 


$$
\rho(U, J, \Pi)=(2 J+1) /\left(48 \sqrt{ } 2 a^{1 / 4} U^{5 / 4}{ }^{3}\right) q(U, J)
$$

is used with

$$
q(U, J)=\exp \left(2\left\{a\left[U-\hbar^{2}(J+1 / 2)^{2} /(2 \theta)\right]^{1 / 2}\right\}\right) .
$$

The moments of inertia $\theta$ an related to the experimental spin cut-off factors $\sigma$ [58] by

$$
\hbar^{2} \sigma^{2}=\theta(U / a)^{1 / 2}
$$

A comparison of this model with different values of $\mathrm{A} / \mathrm{a}$ to data is shown in fig. 1. It turns out that the experimental data indicate a decreasing level-density parameter $a \simeq \mathrm{A} / 7$ for $\varepsilon<3 \mathrm{MeV} / \mathrm{A}$ to $a \simeq \mathrm{A} / 10$, at $\varepsilon \simeq 5 \mathrm{MeV} / \mathrm{A}$ in the case of light nuclei. The results of the method which provides an alternative access to the quantity a complements the information obtained from the study of energy spectra and multiplicities of evaporated light particles in heavy-ion fusion reactions [59].

\section{The Nuclear Level-density Model}

The basic ingredient of the formalism to calculate of is the level density $\rho$ of the highly excited compound nucleus, as a function of the excitation energy $U, \operatorname{spin} J$, parity $\Pi$ and isospin $\mathrm{T}$, and parametrized with various parameters as e.g. the level-density parameter $a$ or its inverse $\mathrm{A} / a$, and the spin-cut off parameter $\sigma$. In other work various theoretical features, especially effects due the finite depth of the mean-field potential [21, 23,60-65], ground state correlations [66], couplings of the single-particle degrees of freedom to collective motion [67] and dynamical polarization [68-73] have been considered.

The level density model discussed here considers the excited nucleus as an isolated system, which confines its constituent $\mathrm{Z}$ protons and $\mathrm{N}$ neutrons for a sufficiently long time. The nuclear states are classified with respect to the 
excitation energies and total quantum numbers by means of the independent (i particle - i hole) proton and (j particle - j hole) neutron configurations. The set of relevant single particle states, comprising the bound and resonant states, is finite due to the finite depth and finite spatial extension of the nuclear potential. The single-particle states are calculated on the basis of a local, spherically symmetric, real nuclear potential $\mathrm{V}(\mathrm{r}, e, \varepsilon)$, with volume and surface components depending on $r$, the single-particle $e$ and the excitation energy $\varepsilon$, parametrized consistent with global results on the nucleon-nucleus potential [68-70] and with theoretical predictions of the radial, single-particle energy and temperature dependence of the effective mass $\mathrm{m}^{*} / \mathrm{m}[66,67,71,72]$ :

$$
V=V_{0}(r)+\left(e-e_{F}\right)(1-n(r, e, \varepsilon))
$$

$\mathrm{V}_{0}(\mathrm{r})$ is a Woods-Saxon potential with depth $\mathrm{V}_{0}+\tau 33(\mathrm{~N}-\mathrm{Z}) / \mathrm{A}(\tau=+1$ for protons and $\tau=1$ for neutrons), reduced radius $r_{0}$, and surface diffuseness â with $e_{\mathrm{F}}$ being. the Fermi energy.

The term $\mathrm{n}(\mathrm{r}, e, \varepsilon)$ is given as

$$
n=[1-a(1-g(e, \varepsilon)) f(r)][1-\beta g(e, \varepsilon) d f(r) / d r]
$$

with

$$
\begin{aligned}
& f=\left[1+\exp \left(\left(r-z A^{1 / 3}\right) / c\right)\right]^{-1} \\
& g=\exp \left(-\varepsilon / \varepsilon_{o}\right) /\left[1+\left(\left(e-e_{F}\right) A^{1 / 3} / 82\right)^{2}\right] .
\end{aligned}
$$

The various parameters $(a, \beta, z, \varepsilon, c)$ are specified in the context of the calculations (see below figs. 2-4). The Z- and N-dependence of the surfacecoupling lengths $\beta$ is adopted from ref. [67]. Spin-orbit terms and the Coulomb potential of the protons are taken in to account.

A discrete single-particle energy $e$ is associated to each resonant single-particle state. Its width $\Gamma_{\mathrm{sp}}$ characterizes the average life time of a nucleon, in this state. For $\hbar / \Gamma_{\mathrm{sp}}$ much larger than the relaxation time for nucleonic degrees of freedom $\left(\simeq 510^{-23} \mathrm{~s}[74]\right)$, such a particle is expected to behave in many respects similarly to nucleons in bound states. On the other hand, excitations corresponding to 
configurations with particles in resonant states can promptly disintegrate by direct emission of nucleons and are not necessarily to be considered to represent states of the excited nucleus. The prompt nucleon emission width $\Gamma_{d}$ of a configuration is equal to the sum of the $\Gamma_{\mathrm{sp}}$ of the populated resonant states. A condition is then imposed that $\Gamma_{d}$ must be smaller than a suitable upper limit $\Gamma_{c}$ $(U)$. As single-particle resonances lying above the effective interaction barrier are some $\mathrm{MeV}$ broad, the set of relevant states consists in fact of bound and quasibound states satisfying $\Gamma_{\mathrm{sp}}<\Gamma_{\mathrm{c}}(U)$. Qualitative consistency of the present leveldensity model with the statistical model of nuclear reactions is achieved by choosing $\Gamma_{\mathrm{c}}(U)<\Gamma(U)$, where $\Gamma(U)$ is the statistical model prediction of the average level-width calculated with (model) level densities satisfying $\Gamma_{d}<\Gamma_{c}(U)$. Empirically it turns out that the procedure to adopt $\Gamma_{\mathrm{c}}(U)$ proportional to the neutron evaporation width [13] normalized to $1 \mathrm{MeV}$ at $\varepsilon<3 \mathrm{MeV} / \mathrm{A}, 1.5 \mathrm{MeV}$ at $\varepsilon=3 \mathrm{MeV} / \mathrm{A}$ and $2 \mathrm{MeV}$ at $\varepsilon=5 \mathrm{MeV} / \mathrm{A}$, for $\mathrm{A}=114-160, \mathrm{~A}=36-40$ and $\mathrm{A}=24-32$ nuclei, respectively, is acceptable.

To compare the predictions of the level-density model with available experimental information of highly excited nuclei $[26-28,59]$ the statistical approach is invoked to evaluate the total state density $\omega(U)$. Following the theory of statistical fluctuations for the grand-canonical ensemble [75], as applied to nuclear level densities $[15,62]$, the distribution of $\Gamma_{d}$ around its average value $\Gamma_{\mathrm{d}^{\mathrm{v}}}{ }^{\mathrm{v}}(\mathrm{U})$ is assumed to be a Gaussian

$$
\omega\left(U, \Gamma_{d}\right)=\omega(U) /\left(2 \operatorname{rog}_{d}{ }^{2}\right)^{1 / 2} \exp \left[-\left(\Gamma_{d}-\Gamma_{d}{ }^{a \nu}\right)^{2} /\left(2 \sigma_{d}{ }^{2}\right)\right]
$$

where $\sigma_{d}(U)$ is the standard deviation of $\Gamma_{d}$ from its average value $\Gamma_{d}^{a v}(U)$ at excitation energy $U$. The quantities $\Gamma_{\mathrm{d}}{ }^{\text {av }}(U)$ and $\sigma_{\mathrm{d}}(U)$ are expressed in terms of single-particle quantities. The nuclear state density $\omega_{\mathrm{cn}}(U)$ (with $\left.\Gamma_{\mathrm{d}}<\Gamma_{\mathrm{d}}(U)\right)$ is approximated by

$$
\left.\omega_{c n}(U) \approx \omega(U) / 2\left\{1+\operatorname{sign}\left(\Gamma_{c}-\Gamma_{d}^{a w}\right) \operatorname{erf}|| \Gamma_{c}-\Gamma_{d}^{a w} \mid /\left(2 \sigma_{d}{ }^{2}\right)^{1 / 2}\right]\right\}
$$

where $\operatorname{sign}(\mathrm{x})$ denotes the sign of $\mathrm{x}$ and $\operatorname{erf}$ is the error function. 
The excitation energy dependence of the parameter $a$ is extracted by comparing the calculated $\omega_{\mathrm{cn}}(U)$ with the Fermi gas expression for the state density

$$
\omega_{c n}(U)=n^{1 / 2} / 12\left(a^{1 / 4} U^{5 / 4}\right) \exp \left(2(a U)^{1 / 2}\right) .
$$

Except for low excitation energies, where the pre-exponential factor in (3.7) is dominant, the parameter $a$ is very close to $\mathrm{S}^{2} / 4 \mathrm{U}$, where $S$ is the nuclear entropy.

At the low excitation energies the level-density parameter $a$ is mainly determined by the properties of the single-particle spectrum around the Fermi energy $e_{\mathrm{F}}$ and by the degree of proton and neutron shell closures. With the parametrization of eqs. 3.1-3.4 values of $a$ are obtained, which are close to those resulting from calculations using an $e, \varepsilon$-independent nuclear potential term $\mathrm{V}_{\mathrm{o}}(\mathrm{r})$. In figs. 2 to 4 we show results of our calculations for the inverse level density for heavy and medium heavy nuclei. As is seen in these figures, the trend of the shell effects in experimental systematics of $a$ in the neutron resonance region [76], is reasonably well reproduced (compare results for $208 \mathrm{~Pb}$ and $198 \mathrm{Pt}$ (fig. 2), $40 \mathrm{Ca}$ and $35 \mathrm{Cl}$ (fig. 3) ). At very high excitation a rather reflects global characteristics of the energy (and width) distribution of the finite number of available proton and neutron single-particle states $[15,62,78]$ and of the leveldensity model consistency condition $\Gamma_{d}<\Gamma_{c}(U)$. For $\varepsilon \gg \varepsilon_{0}$ and $g(e, \varepsilon) \simeq 0$ the results approach those of calculations with the $\varepsilon$-independent potential $\mathrm{V}_{\mathrm{o}}(\mathrm{r})+\left(e-e_{\mathrm{F}}\right)(1-\mathrm{a} f(\mathrm{r}))$ and with $\mathrm{m}^{*} / \mathrm{m}=1-\mathrm{a} f(\mathrm{r}) \leqq 1$. However, the predictions of the model are restricted to $\varepsilon \leqq 3 \mathrm{MeV} / \mathrm{A}$ for $\mathrm{A} \geqq 114, \varepsilon \leqq 5 \mathrm{MeV} / \mathrm{A}$ for $\mathrm{A}=24-32$ nuclei, where strong experimental evidence for equilibration in highly excited nuclei exists [26-28], [11, 16, 17]. For nuclei between closed proton and neutron shells, $a(\varepsilon)$ is steadily decreasing with increasing $\varepsilon$ (figs. 2-4). At the same value of $\varepsilon$ and for the same set of potential parameters $\mathrm{A} / \mathrm{a}$ is generally decreasing with decreasing A [73]. Nevertheless, an additional dependence of $a$ on N-Z is observed. Dynamical effects enhance $a$ at moderate excitations, as long as the number of single-particle levels increases on both sides of $e_{\mathrm{F}}$, The increase of $\Gamma_{\mathrm{sp}}$ 
of the quasi-bound levels leads to a further reduction of $a$ at high excitation (see results for $160 \mathrm{~Tb}$ in fig. 2). A realistic description of the $e$, $\varepsilon$-dependence of the mean potential leads to better agreement with data from refs. 26-28, especially in the transition region around 1.2 MeV/A. For $\mathrm{In}, \mathrm{Sn}, \mathrm{Sb}$ isotopes around the $\mathrm{Z}=50$ magic number the calculations result in a nearly constant value $a=\mathrm{A} /(8.5-9)$ for $\varepsilon \leqq 0.7 \mathrm{MeV} / \mathrm{A}$, while the general trend of decreasing $a$ starts at higher $\varepsilon$ values. Significant shell effects exist for nuclei close to doubly-magic nuclei, especially for $208 \mathrm{~Pb}$. In fig. 2 the curves referring to $208 \mathrm{~Pb}$ and $198 \mathrm{Pt}$ approach the region of values of $a(\varepsilon)$ for $(\mathrm{Z}, \mathrm{N})=(77-88,120-132)$ nuclei. For $208 \mathrm{~Pb}, a$ increases from $\simeq \mathrm{A} / 20$ at low excitation [76] to about $\mathrm{A} / 12$ at about $0.5 \mathrm{MeV} / \mathrm{A}$, while its decrease at higher excitations depends on dynamical effects, which are represented in $\mathrm{V}$ of eqs. (3.1-3.4) by the term $g(\varepsilon)$.

Several potential parameter sets have been used in calculations for $A=35-40$ nuclei. Although the values of $a(\varepsilon)$ for a specified nucleus slightly depend on the particular parameter set, the trend of the results is similar in all cases. While for $\mathrm{A} \simeq 160$ nuclei at $\varepsilon \approx 3 \mathrm{MeV} / \mathrm{A}$ three neutron and 10 proton-quasi-bound singleparticle levels satisfy $\Gamma_{\mathrm{sp}}<\Gamma_{\mathrm{c}}(\varepsilon=3 \mathrm{MeV} / \mathrm{A})$, for $\mathrm{A}=40$ nuclei only two neutron and two proton-quasi-bound single-particle levels must be taken into account. The strong reduction of the number of relevant (especially proton) resonant single-particle levels induces a weak dependence of $a$ on $\Gamma_{c}(\varepsilon)$ for light nuclei. Agreement is found for the present calculations for non-closed shell $A=40$ nuclei (e.g. $35 \mathrm{Cl}$ ) with the experimental result $a=\mathrm{A} / 8$ for $\varepsilon \leqq 1.7 \mathrm{MeV} / \mathrm{A}$ [59] and with theoretical results [73] (fig. 3). Simplified calculations for 40Ca, using a particular set of experimental bound and (discrete) resonant single-particle levels from ref. 77 and ignoring the restrictions $\Gamma_{\mathrm{sp}}, \Gamma_{\mathrm{d}}<\Gamma_{\mathrm{c}}(U)$, lead to $a(\varepsilon)$ values fairly close to those of the complete model, but the decrease of $a$ occurs at excitation energy beyond $3 \mathrm{MeV} / \mathrm{A}$ (fig. 3 ). 


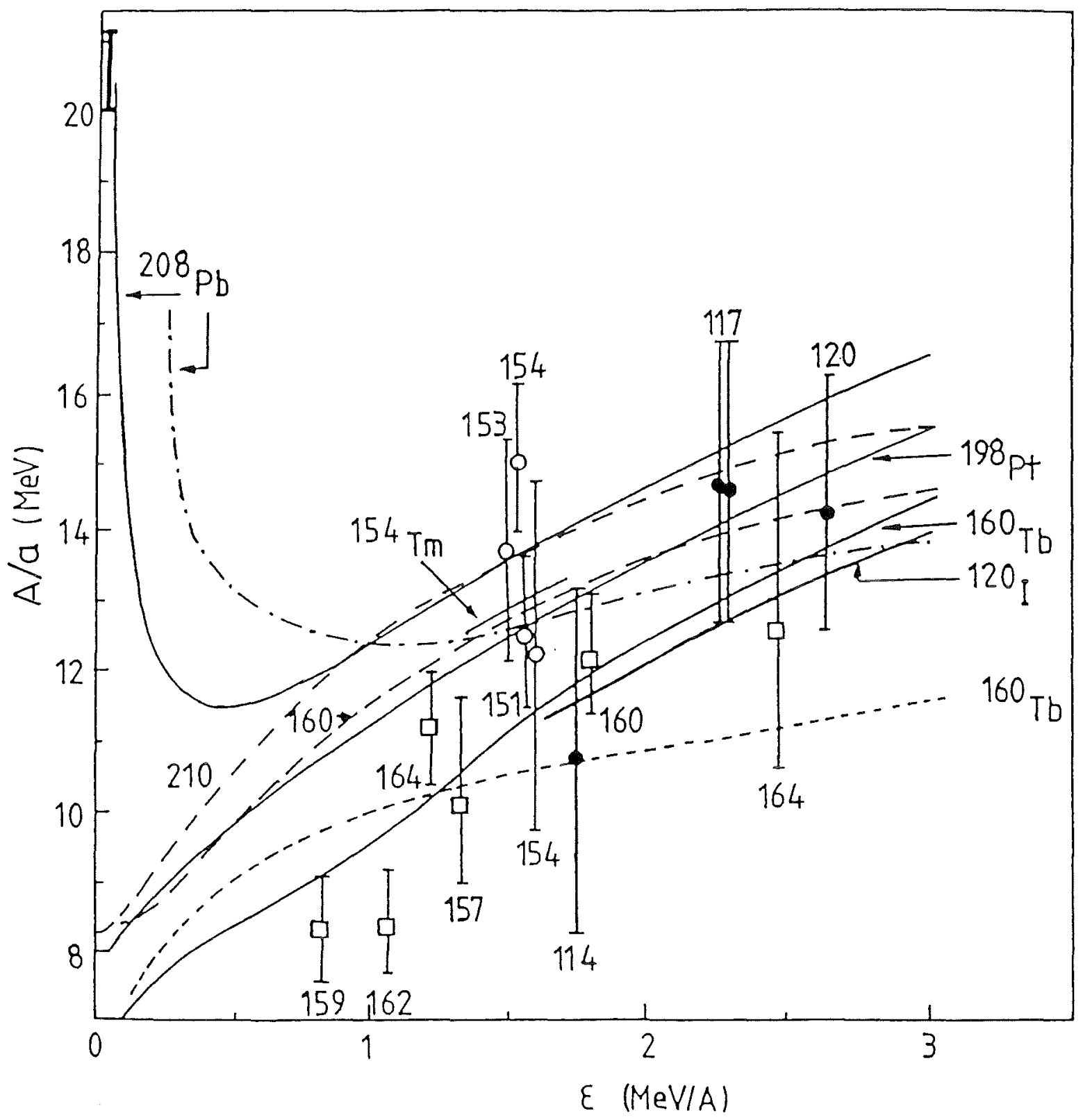

Fig. 2: Energy dependence of the inverse level-density parameter A/a calculated in the framework of the present model for various mass numbers $A$ :

Parameter values:

$V_{0}=51 \mathrm{MeV}, r_{0}=1.26 \mathrm{fm}, \hat{a}=0.67 \mathrm{fm}, z=1.15 \mathrm{fm}, c=0.6 \mathrm{fm}, \mathrm{a}=0.36$, $\varepsilon_{o}=1.6 \mathrm{MeV} / \mathrm{A}$ (for $\left.A=198-208\right)$ and $\varepsilon_{o}=1.05+28 / \mathrm{A} 2 / 3$ (for $\left.A=114-164\right), \Gamma_{c}(\varepsilon=3$ $M e V / A)=1 \mathrm{MeV}$. The results of this model (solid lines) are compared to experimental data from refs. 26, 27, 28 and 78 and various theoretical calculations: Calculations neglecting the second, $(e, \varepsilon)$ - dependent term in $V[15,63]$ (short dash); from ref. 73 (long dash); $a=S^{2} / 4 U$ [23](dash-dot). 


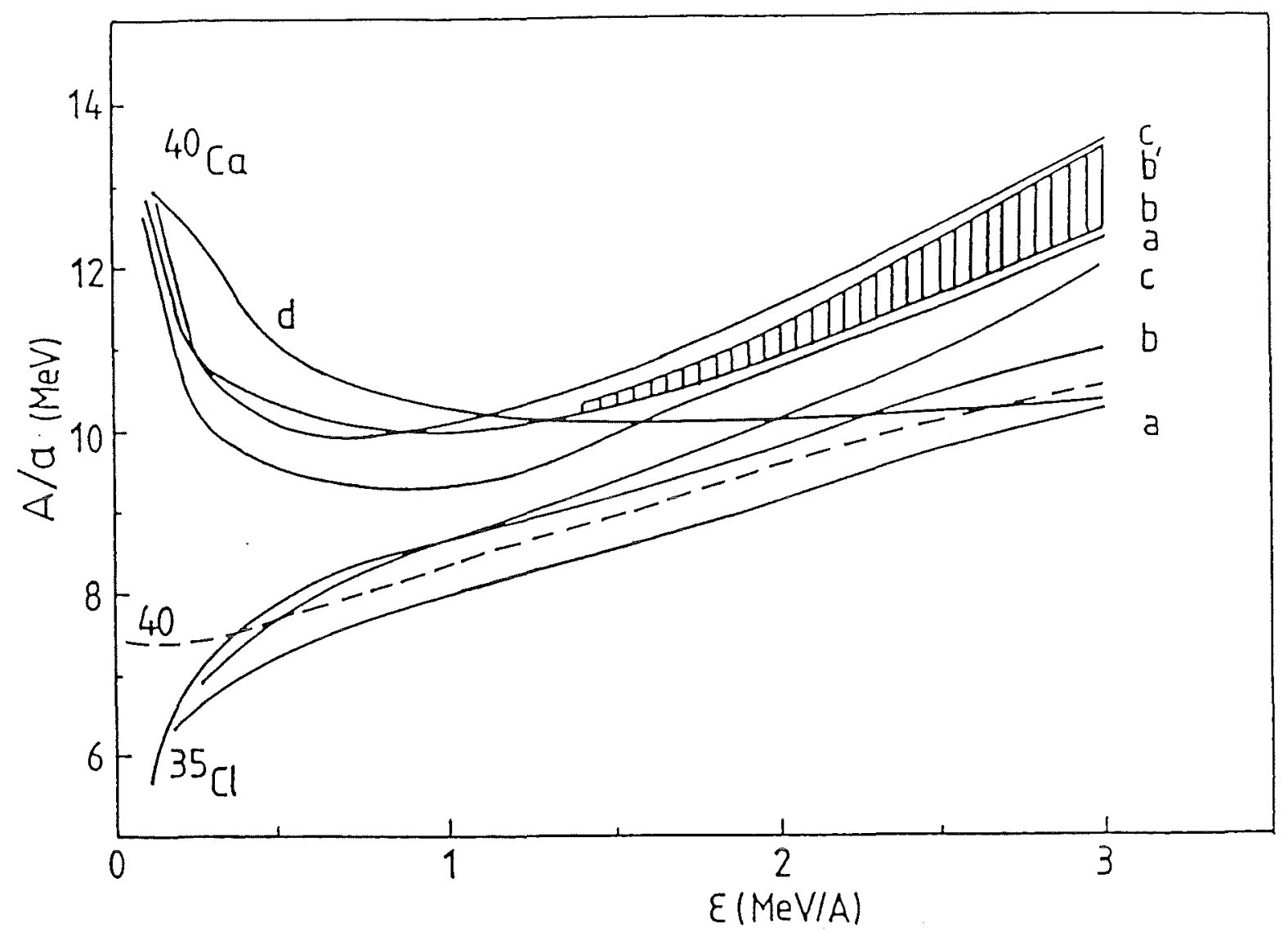

Fig. 3: Energy dependence of the inverse level-density parameter $\mathrm{A} / \mathrm{a}$ of $35 \mathrm{Cl}$ and ${ }^{40} \mathrm{Ca}$ given by the present model (solid lines) with different parameter values. The common parameter values are: $\varepsilon_{0}=5.4 \mathrm{MeV} / \mathrm{A}, \Gamma_{c}(\varepsilon=3 \mathrm{MeV} / \mathrm{A})=1.5 \mathrm{MeV}$. For different curves:

a. $V_{0}=51 \mathrm{MeV}, r_{0}=1.26 \mathrm{fm}, \hat{a}=0.67 \mathrm{fm}, z=1.15 \mathrm{fm}, c=0.6 \mathrm{fm}, \mathrm{a}=0.36$;

b. $V_{0}=58.8 \mathrm{MeV}, r_{o}=1.18 \mathrm{fm}, \hat{a}=0.7 \mathrm{fm}, z=1.4 \mathrm{fm}, c=0.2 \mathrm{fm}, \mathrm{a}=0.36$;

c. like b. with $\mathrm{a}=0.45$;

$b^{\prime}$. like b. with $\Gamma_{c}(\varepsilon=3 \mathrm{MeV} / \mathrm{A})=0.3 \mathrm{MeV}$;

d. Results with bound and (discrete) resonant levels taken from ref. 77.

The predictions of the present level-density model are consistent with the trend of $a(\varepsilon)$ for $\mathrm{A}=24-36$ nuclei with $\varepsilon \leqq 5 \mathrm{MeV} / \mathrm{A}$, inferred from total fusion excitation functions, and with level-density parameters at low excitation energies from statistical model calculations for the $14 \mathrm{~N}, 16,18 \mathrm{O}+12 \mathrm{C}$ reactions [79]. 


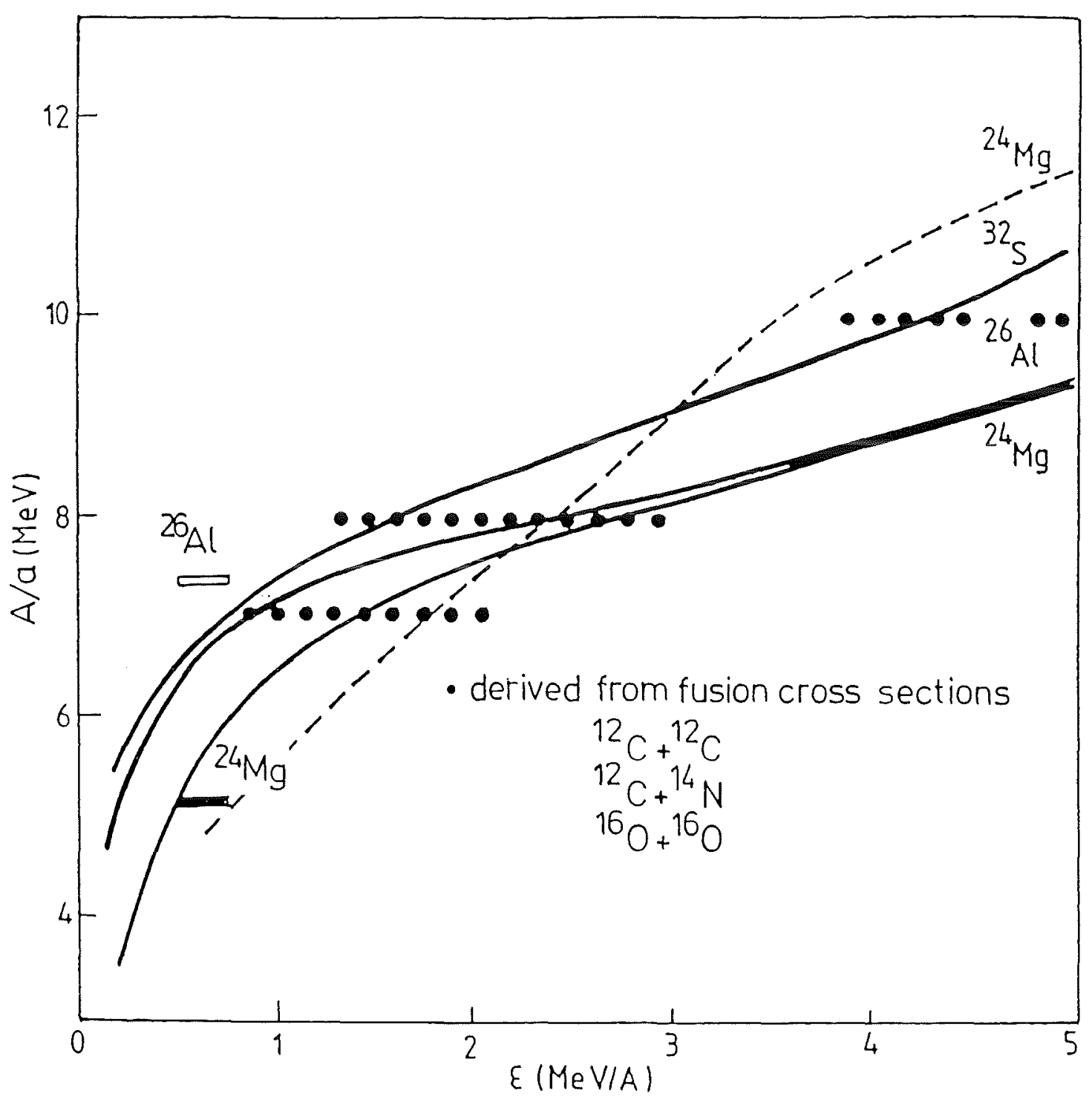

Fig. 4: Energy dependence of the inverse level-density parameter A/a of $24 \mathrm{Mg}$, $26 \mathrm{Al}$ and $32 \mathrm{~S}$, calculated within the present level-density model (dashed line). Parameter values:

$V_{0}=58.8 \mathrm{MeV}, r_{o}=1.18 \mathrm{fm}, \hat{a}=0.7 \mathrm{fm}, z=1.4 \mathrm{fm}, c=0.6 \mathrm{fm}, \mathrm{a}=0.45, \varepsilon_{o}=6.4$ $\mathrm{MeV} / \mathrm{A}, \Gamma(\varepsilon=5 \mathrm{MeV} / \mathrm{A})=2 \mathrm{MeV}$.

The calculations shown by solid lines result from the set of bound and quasibound single-particle levels recommended in ref. 15. The (simplified model) values represented by rectangles are taken from ref. 79 . 
The gross features of $\rho(U, J)$ of light nuclei can be described in a simplified model [15]. It is based on a set of single-particle states deduced from experimental results and complemented with deeply lying levels calculated with a WoodsSaxon potential $\left(\mathrm{V}_{0}=51 \mathrm{MeV}, \mathrm{r}_{0}=1.27 \mathrm{fm}, \hat{\mathrm{a}}=0.67 \mathrm{fm}\right)$. The set of single-particle levels is restricted to the $1 \mathrm{~s}_{1 / 2}$ to $2 \mathrm{p}_{3 / 2}$ levels for $24 \mathrm{Mg}$ and $26 \mathrm{Al}$, and to $1 \mathrm{~s}_{1 / 2}$ to $2 \mathrm{p}_{1 / 2}$ levels with the condition $\Gamma_{\mathrm{sp}}<1 \mathrm{MeV}$. The calculated level density $\rho(U)$ is shifted on the U-scale by $\Delta=5 \mathrm{MeV}(24 \mathrm{Mg}), 1 \mathrm{MeV}(26 \mathrm{Al})$ and $4 \mathrm{MeV}(35 \mathrm{~S})$, respectively in order to obtain a better agreement with data at $\varepsilon \leqq 1 \mathrm{MeV} / \mathrm{A}$. This empirical procedure takes in to account phenomenologically the effects of residual interactions. The result of this simplified description is also displayed in fig. 4 . The calculated spin cut-off factors $\sigma(U)$ are in fair agreement with phenomenologically derived values (fig. 5).

Due to specific features of the statistical approach to level densities, a grand canonical ensemble is used for a nucleus in such a way, that $N, Z, U, M$ are the averages and most probable values of the relevant distributions. For light systems the value of the fluctuations of these quantities is a considerable fraction of their average values, and interesting details of the level densities are smoothed out $[15,62]$. In addition it has been shown that the usual assumption of a uniform distribution of parity, based on the statistical random-coupling argument [13], does not apply to light nuclei with $A \leqq 40[15,38]$. These limitations of the statistical approach have been avoided by exact combinatorial (micro-canonical) level-density calculations. For that purpose the combinatorial method and the computer code elaborated in ref. 15 have been used. Advantage is taken of the fact that the simplified level-density model gives an acceptable description of the gross properties of $\rho(U, J)$ of light nuclei so that large-scale combinatorial calculations can be performed on the basis of it. This fact drastically reduces the computational effort. 


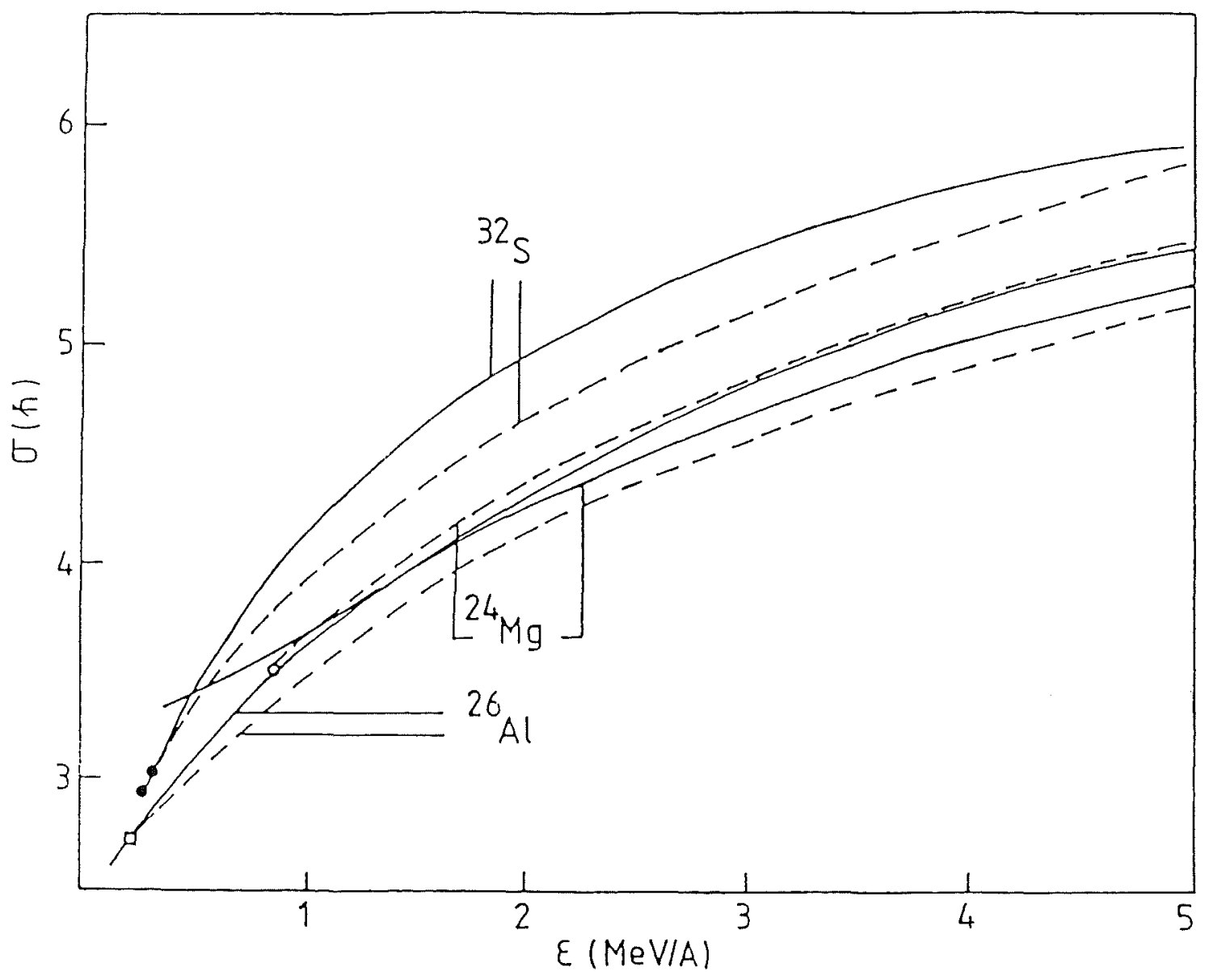

Fig. 5: Values of the spin cut-off parameter $\sigma$. The results displayed by the solid lines correspond to the calculations shown in fig. 4. The dashed lines are deduced from experimental values (using eqs. 2.16-2.17).

For each nucleus $(24 \mathrm{Mg}, 26 \mathrm{Al}, 32 \mathrm{~S})$ and for given values of $\Pi, \mathrm{T}$ a two-dimensional matrix $p([U], J, \Pi, T)$ has been constructed. The quantity $[U]$ is the integer part of $\mathrm{U}+0.5 \mathrm{MeV}$ with $\mathrm{U} \leqq 5 \mathrm{~A} \mathrm{MeV}$. The contour lines of $\rho$ ( $[U], \mathrm{J}, \Pi=+1, \mathrm{~T}=0$ ) of ${ }^{24} \mathrm{Mg}$ (fig. 6) illustrate the typical features of the predicted level densities of highly excited light nuclei. Gross structures, about $20 \mathrm{MeV}$ broad, are evident. A 


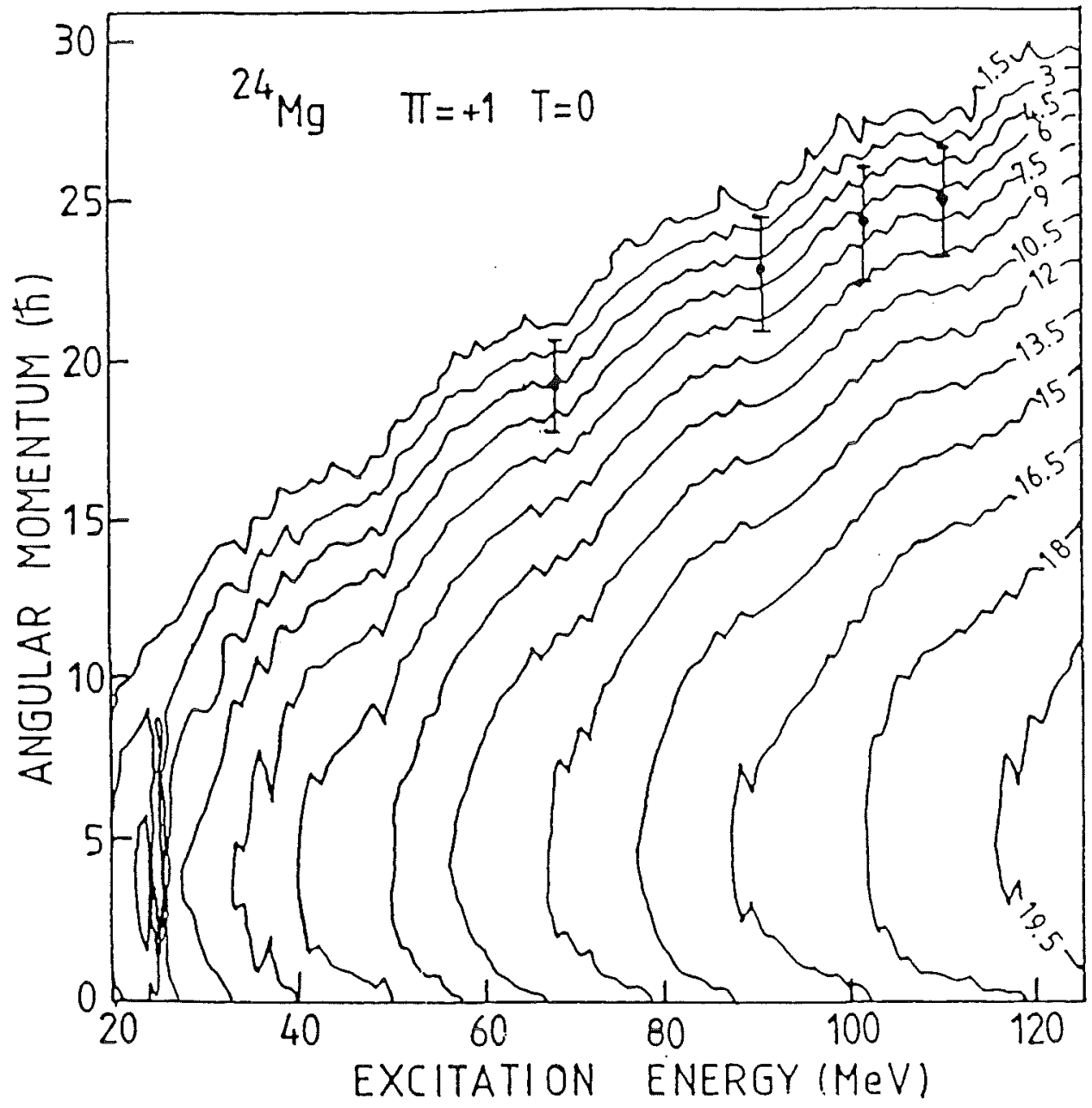

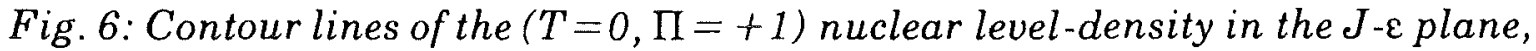
as derived for $24 \mathrm{Mg}$ with a set of single-particle states corresponding to the results displayed in figs. 4 and 5 (solid line). The experimental values of $J_{c r}$ indicated are from data [16] by using the relation $\sigma_{F}=n / \mathrm{k}^{2}\left(J_{c r}+1\right)^{2}$.

fine structure, a few $\mathrm{MeV}$ broad, is superimposed. Both gross and fine structure of the different contour lines are remarkably well correlated in energy. 


\section{Fusion Excitation Functions}

The calculations were performed for $12 \mathrm{C}+12 \mathrm{C}, 12 \mathrm{C}+14 \mathrm{~N}, 10 \mathrm{~B}+14 \mathrm{~N}$ and $160+160$ total fusion cross section excitation functions over the broad energy range $\mathrm{E}_{\mathrm{cm}} \leqq 120 \mathrm{MeV}$ using realistic combinatorial level densities $\rho(U, J, \Pi, \mathrm{T})$ for $24 \mathrm{Mg}, 26 \mathrm{Al}$ and $32 \mathrm{~S}$ from our model (sect. 3) for $\varepsilon \leqq 5 \mathrm{MeV} / \mathrm{A}$. Assuming isospin conservation only compound nucleus levels with $\mathrm{T}=0$ are involved. The results are given in figs. 7 to 9 .

The strong selectivity due to conserved quantum numbers for the $12 \mathrm{C}+12 \mathrm{C}$ and $160+160$ systems of identical spin-zero ions leads to an extreme sensitivity of the fusion cross section to details of the compound nucleus level-density. Structures in experimental $12 \mathrm{C}+12 \mathrm{C}, 16 \mathrm{O}+16 \mathrm{O}$ fusion cross sections appear to be correlated to structures of in $\rho([U], J, \Pi=+1, T=0)$ of $24 \mathrm{Mg}, 32 \mathrm{~S}$ for $\mathrm{E}_{\mathrm{cm}} \leqq 12$ $35 \mathrm{MeV}$, and 18-35 MeV, respectively. The calculations with level densities deduced from the model globally reproduce the fusion cross section structures. However, the amplitude of oscillations overestimated. This is due to the neglect of residual interactions in the model leading to too high degeneracy and is corrected by an appropriate redistribution of the model levels.

The smoothed level density $\rho_{\mathrm{s}}$ resulting from such procedure retains the main features of the $U$ dependence of the exact combinatorial $\rho$, but it is less dependent on artefacts arising from the simplification of the nuclear model. The calculations with $\rho_{\mathrm{s}}([U], \mathrm{J}, \Pi=+1, \mathrm{~T}=0)$ reproduce the oscillating $12 \mathrm{C}+12 \mathrm{C}[31$, $34]$ and $160+160$ [32-34] fusion excitation functions within the experimental uncertainties (figs. 7). The good agreement with data from ref. 32 is shown in fig. 7b. The fusion cross section oscillations are very sensitive to a change of the shifts $\Delta$ (defined in sect. 3). A variation of $\Delta$ by $1-2 \mathrm{MeV}$ deteriorates the agreement with the data.

The trend of $12 \mathrm{C}+12 \mathrm{C}[16,34]$ and $160+160[60]$ total fusion excitation functions, found in measurements with large energy steps and in quantum 

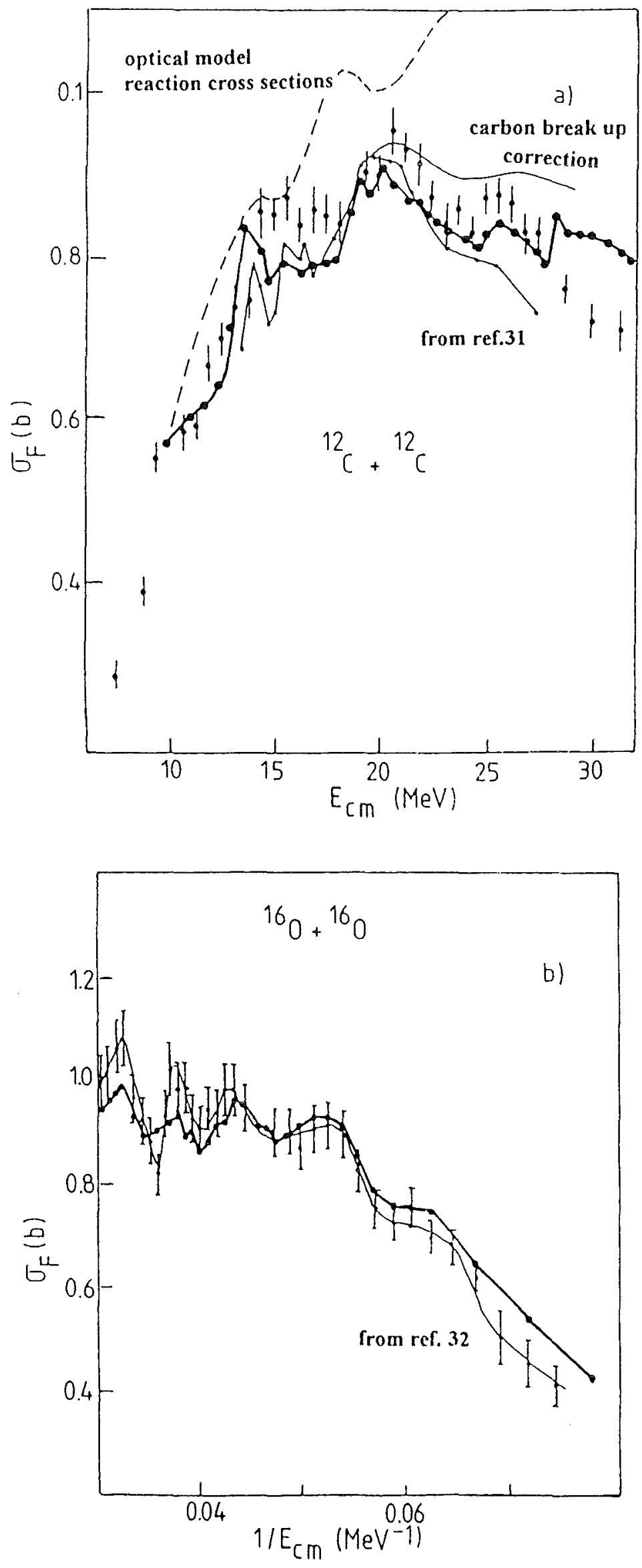

Fig. 7: Total fusion cross sections for $12 C+12 C$ (a) and $160+16 O(b):$

Theoretical results using combinatorial compoundnucleus level densities (fat solid line with dots) compared to the following experimental results:

(a) ref [34] (dots with error bars; ref [31] (thin line with dots) and including $12 C+3 \mathrm{a}$ breakup correction (thin line). (b) ref. [32] (dots with error bars connected by thin line). For ${ }^{12} \mathrm{C}+12 \mathrm{C}$ also the optical model reaction cross section is given. 


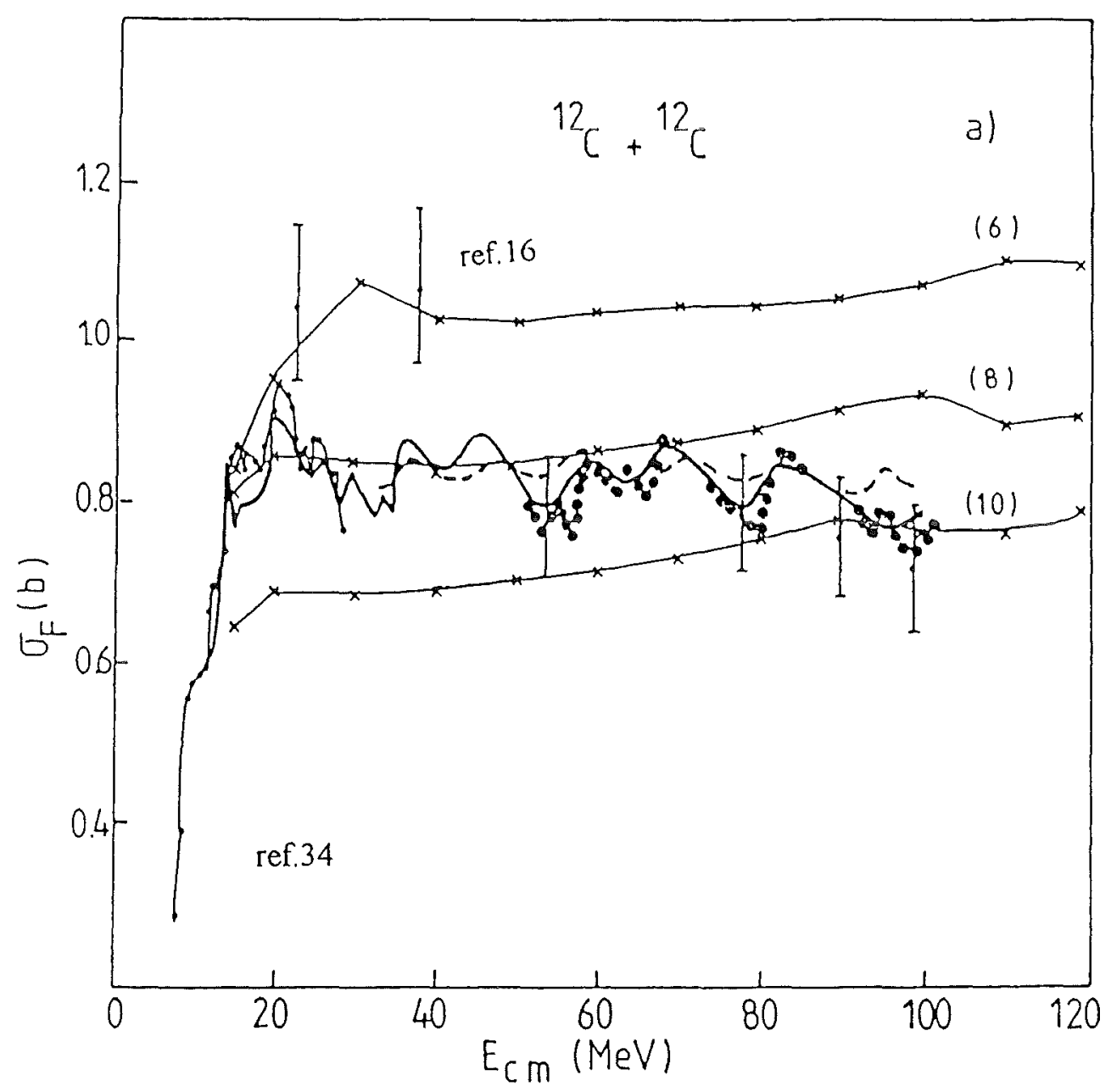

Fig. 8a: Total fusion cross sections excitations functions for $12 C+12 C$, calculated within the present fusion model using combinatorial compound-nucleus level densities $\mathrm{\rho}(U, J, \Pi=+1, T=0)$ and compared to experimental results from ref. [16] (dots with error bars) and [34] (dots on thin line). The fat solid line represents calculations with a smoothed $\rho$, the dashed line with $\rho(U, J, \Pi=+1$, $T=0)=1 / 2 \rho(U, J, T=0)$. Full dots represent calculations with unsmoothed $\rho$. The thin lines with crosses are calculations like in fig. 1 with the label indicating the values of the inverse level-density parameter.

molecular dynamic calculations of $160+160$ fusion cross sections at $E_{c m}=90$, $110 \mathrm{MeV}$ [61], is reproduced by the present calculations (figs. 8 a, b). About 20 $\mathrm{MeV}$ broad gross structures with about $100 \mathrm{mb}$ peak-to-valley differences are predicted over the whole energy range. Assuming parity to be uniformly 


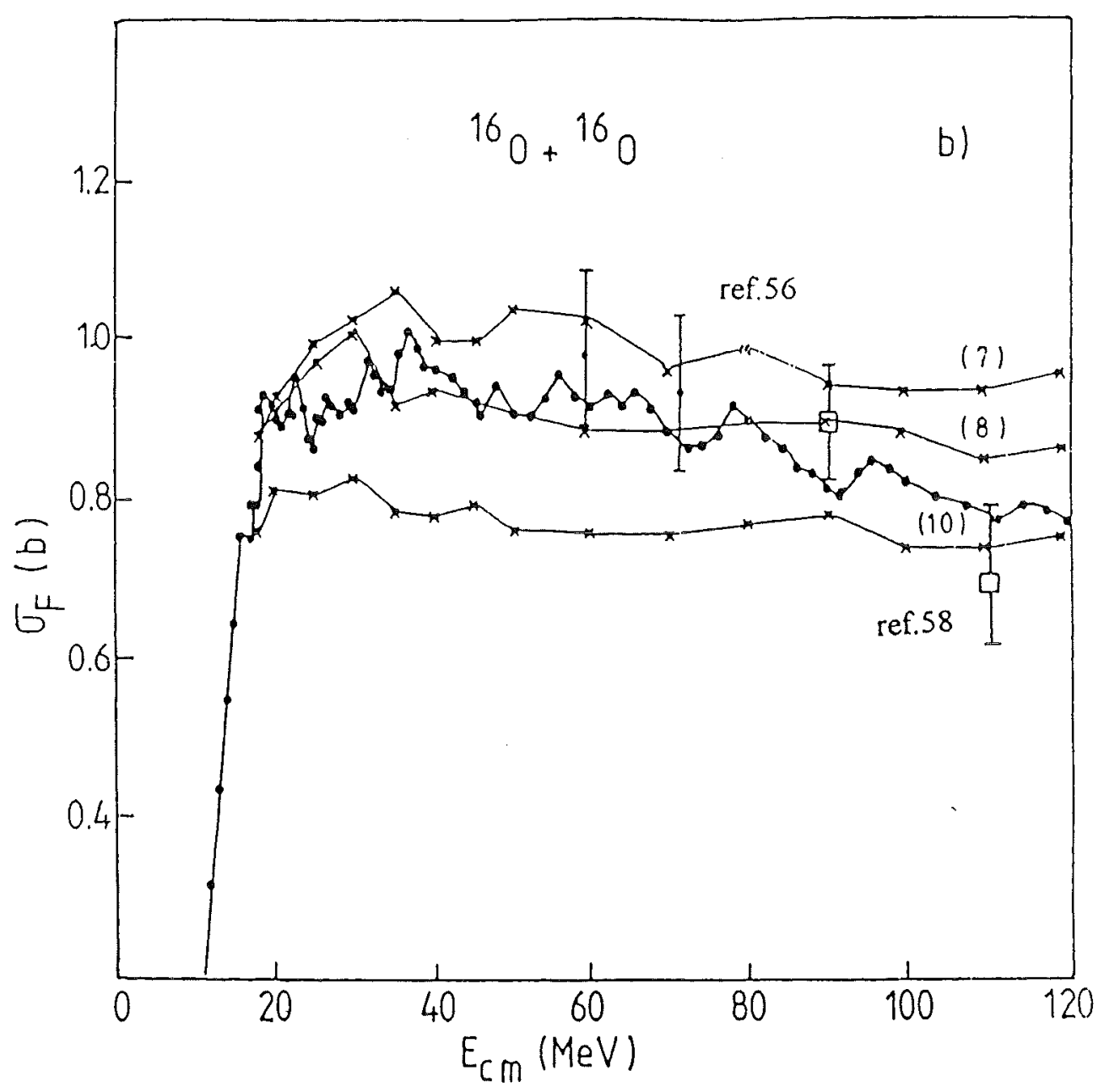

Fig. 8b: Total fusion cross sections excitations functions for $160+16 O$, calculated within the present fusion model as in fig. 8 a (dots on fat line, unsmoothed $\rho$ ). They are compared to experimental results ([56] dots) and to calculated values of $\sigma_{F}$ ([58], squares).

distributed, i.e. $\rho_{\mathrm{s}}([U], J, \Pi=+1, T=0)=1 / 2 \rho_{\mathrm{s}}([U], J, T=0)$, significantly changes the oscillation pattern in $\sigma_{F}$. The gross structures disappear and the oscillations are weakened.

For spin-zero non-symmetric ions, even and odd $l$ partial waves are present in the entrance-channel and compound-nucleus states with parity $\Pi=(-1)^{l} \Pi_{1} \Pi_{2}$, and angular momentum $J=l$ are populated from the $l$ partial wave. For non-zero 
channel spin $s$, the $l$ partial wave populates compound-nucleus states with $\Pi=(-1)^{l} \Pi_{1} \Pi_{2}$, but different $J=|l-s|, \ldots ., l+s$. In addition compound-nucleus states with $J$ and $\Pi=-1,+1$ are fed from neighbouring partial waves. In this case, the fusion cross section exhibits the structures of the compound-nucleus level density in a less pronounced way.

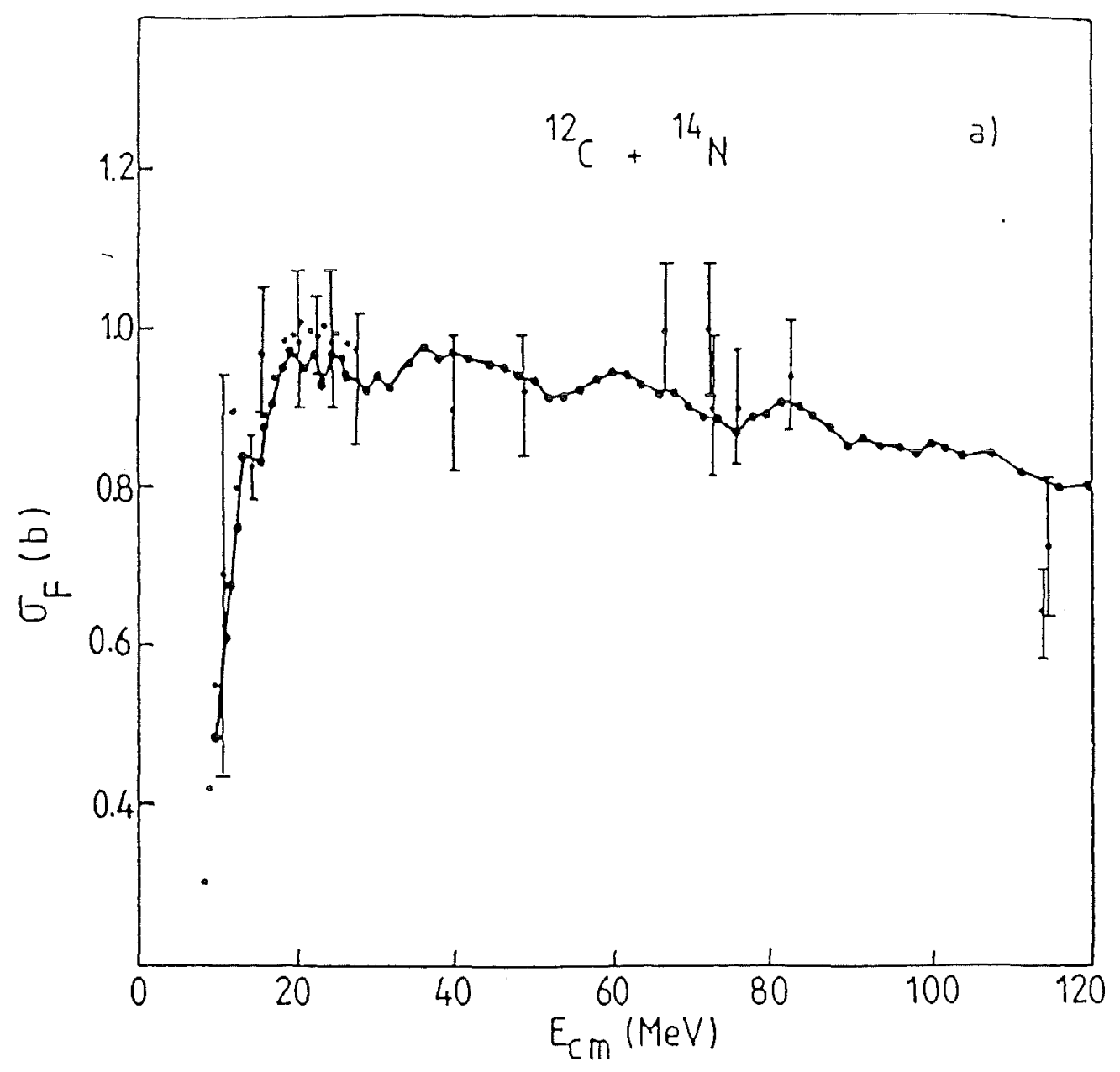

Fig. 9a: $12 C+14 N$ total fusion cross section calculated within the present fusion model, using combinatorial compound-nucleus level densities (fat dots on line), and compared to experimental results $[11,17,31,34,55]$.

Fig. 9a displays the calculated $12 \mathrm{C}+14 \mathrm{~N}$ fusion excitation cross section and shows agreement with data $[11,17,31,34,55]$. The weak gross structure reflects the structure in the level-density of $26 \mathrm{Al}$ and is also seen in the calculation of the $10 \mathrm{~B}+160$ case (fig. 9 b). However, when taking into account the channel spin 


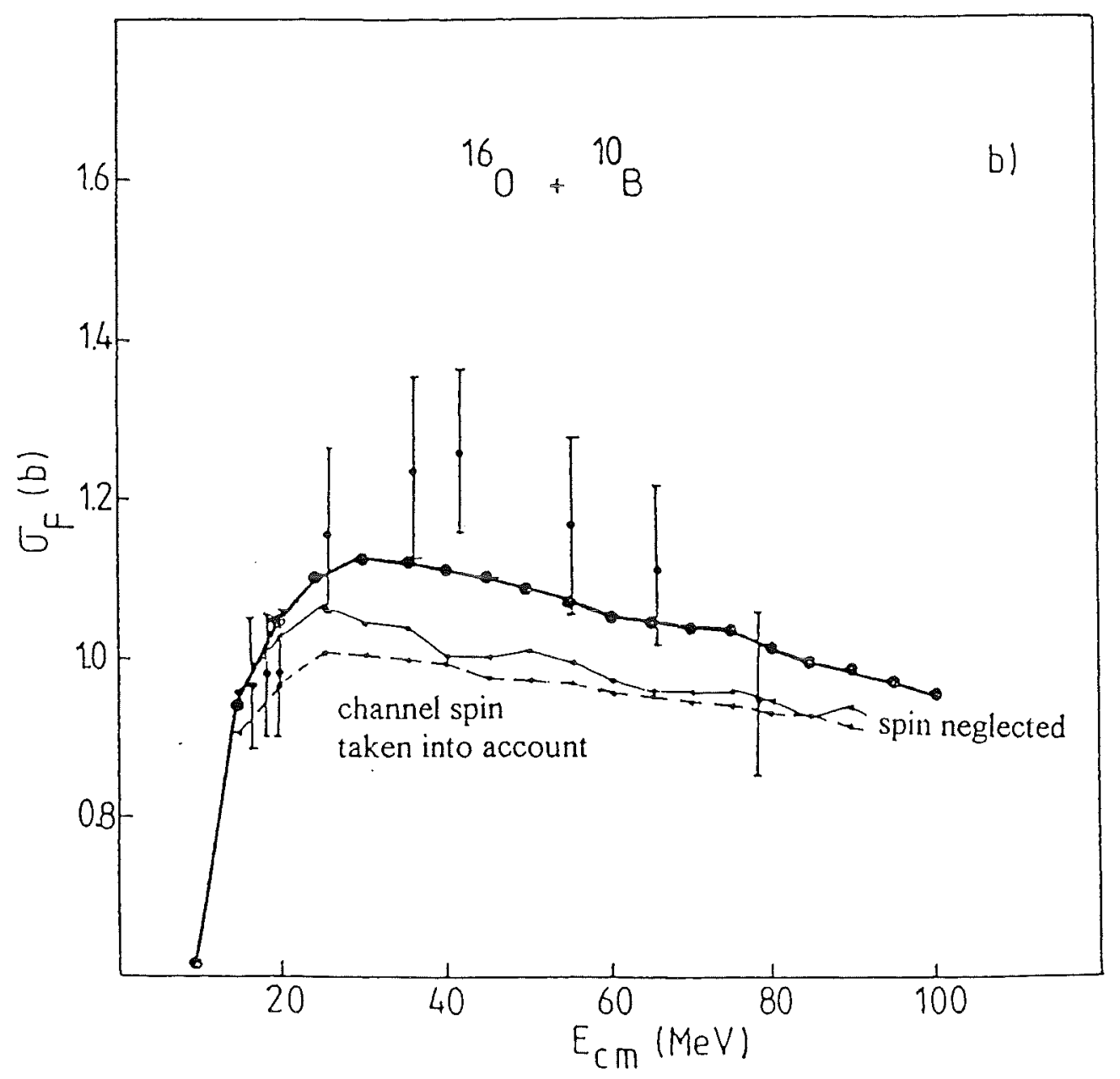

Fig. 9b: $16 O+10 B$ total fusion cross section calculated within the present fusion model and compared to experimental results [11]. Calculation are without (dots on thin line) and with (dots on dashed line) inclusion of the spin of 10B. A peripheral nonelastic fusion component added to the volume part of $W_{F}$ improves the agreement of the calculations (fat dotted line) with the data.

$s=3 \hbar$ the structure is further smoothed, and the fusion excitation function becomes insensitive to details of the level density of the highly excited compoundnucleus.

The theoretical $10 \mathrm{~B}+16 \mathrm{O}$ fusion cross section considerably underestimates the experimental results [11]. In fact, the $10 \mathrm{~B}+16 \mathrm{O}$ system exhibits several peculiarities as compared to the cases shown in figs. 7 and 8 . The nucleus $10 \mathrm{~B}$ has an increased number of low-lying excited states, the $10 \mathrm{~B}+160$ channel has a 
higher Q-value for compound-nucleus formation, and there is a strong absorption of partial waves with large $l$ due to direct couplings to numerous well-matched non-elastic channels. This is reflected in the empirical optical potential with a comparatively strong energy variation of $\mathrm{dW} / \mathrm{dE} \mathrm{cm}_{\mathrm{c}}=-0.31$.

According to eqs. $2.3-2.11$, a good matching between the entrance-channel and many non-elastic channels for $J \geqq J_{c r}(U)$ leads to strong absorption, and increases the absorption by $\mathrm{W}^{\mathrm{F}}$ for $J \leqq J_{y . l}$. too, due to non-negligeable contributions of terms $B$ (and $C$ ) in addition to $A$ in eq. (2.6). The absorption of a part of the flux from the entrance-channel into non-elastic-channels due to direct coupling enhances the total fusion cross section. In the actual calculations contributions to $F$ of eq. (2.14) due to terms $B$ (and $C$ ) of surface type are simulated as the difference between $F$ for $\mathrm{r}_{\mathrm{F}}=1.6 \mathrm{fm}, \mathrm{k}=510^{-3}$ and $\mathrm{r}_{\mathrm{F}}=1.35 \mathrm{fm} \mathrm{x}=510^{-3}$. The improved agreement with $10 \mathrm{~B}+160$ total fusion data [11] is shown in fig. $9 \mathrm{~b}$.

\section{Conclusions}

The present optical model description of light heavy-ion fusion takes into account properties of the compound-nucleus, characteristics of the entrance-channel and of the reaction dynamics, in a way consistent with general constraints of the unified theory of nuclear reactions. The basic quantities in the expression of the fusion cross section (and the fusion transmission coefficients) are the imaginary fusion potential and the wave functions generated by the total optical potential in the entrance-channel.

Over a broad energy range, a competition between direct processes and total fusion of approximately mass-symmetric light systems mainly occurs for angular momenta corresponding to the relatively low level-density region on the left hand side of the Yrast line of the compound-nucleus. In this paper the compoundnucleus effects in the formal expression of the entrance-channel optical potential 
are treated in first-order approximation. This leads to a proportionality of the imaginary fusion potential with both the level density of the compound-nucleus and with the sum of dynamical terms which determine the relative importance of elastic and non-elastic fusion mechanisms. These depend on entrance-channel characteristics, i.e. the direct coupling-strength and the matching with nonelastic channels.

While the elastic-fusion dynamical term seems to be reasonably well described in a macroscopic way, the non-elastic fusion terms require further consideration of the direct reaction dynamics and the nuclear structure of the contributing nonelastic channels.

$12 \mathrm{C}+12 \mathrm{C}, 12 \mathrm{C}+14 \mathrm{~N}, 10 \mathrm{~B}+16 \mathrm{O}$ and $16 \mathrm{O}+16 \mathrm{O}$ total fusion excitation functions have been calculated for $\mathrm{E}_{\mathrm{cm}} \leqq 120 \mathrm{MeV}$. Both the total optical potential and the dynamical fusion terms are treated in a phenomenological procedure. The analysis of the trend of experimental fusion excitation functions using classical level-density expressions reveals clear evidence for a decrease of the leveldensity parameter from $a \simeq \mathrm{A} / 7$ for $\varepsilon \simeq 3 \mathrm{MeV} / \mathrm{A}$ to $a \simeq \mathrm{A} / 10$ at $\varepsilon=5 \mathrm{MeV} / \mathrm{A}$ for $\mathrm{A}=24-32$ nuclei,.

Special attention has been paid to the analysis of the relation between the properties of light heavy-ion fusion cross sections and specific properties of the level density of highly excited light compound-nuclei. A realistic level-density model, taking into account the finiteness and the excitation energy dependence of the mean-field nuclear potential, and the finite life-time of the highly excited compound-nucleus, gives a reasonable description of experimental data. Large scale combinatorial calculations of excitation energy, angular momentum, parity and isospin dependent level densities of $24 \mathrm{Mg}, 26 \mathrm{Al}$ and $32 \mathrm{~S}$ at excitations below $5 \mathrm{MeV} / \mathrm{A}$ were performed on the basis of a simplified, nevertheless realistic version of the level-density model. Effects of residual interactions are phenomenologically taken into account. It is found that parity is not uniformly 
distributed and structures are present in the calculated micro-canonical level density of highly excited light nuclei.

Fusion cross section calculations which treat exactly angular momentum, parity and isospin conservation, and use combinatorial level densities give a good description of both global and detailed properties of experimental fusion excitation functions. The fusion oscillations of the special $12 \mathrm{C}+12 \mathrm{C}$ and $160+160$ systems with spin-zero identical ions are well reproduced. Distinct oscillations, correlated with the structures in the level density of the compoundnucleus, are predicted at high energies. These structures are weakened, but remain in the calculated fusion excitation function of $12 \mathrm{C}+14 \mathrm{~N}$. The rather large channel spin of the $10 \mathrm{~B}+16 \mathrm{O}$ system makes the fusion excitation function insensitive to the structures in the level density of the $26 \mathrm{Al}$ compound nucleus.

The anomalous behaviour of the $10 \mathrm{~B}+160$ fusion cross sections at moderate energies is attributed to a substantial contribution of non-elastic fusion, consistent with specific entrance-channel properties of this system.

A microscopic derivation of the radial, angular momentum and energy dependence of the dynamical fusion terms and a simultaneous description of elastic scattering, total fusion and direct cross sections over a broad energy range, with better consistency within a detailed optical model formalism, are desirable. This would lead to a precise treatment of the fusion dynamics. Then, more accurate information on the properties of highly excited light nuclei at high angular momenta could be obtained from the detailed study of fusion cross section excitation functions. 


\section{References}

1. J.B. Natowitz, Phys. Rev. C1, 623 (1970)

2. J.B. Natowitz, E.T. Chulick, M.N. Namboodiri, Phys. Rev. C6, 2133 (1972)

3 J. Galin, D. Guerreau, M. Lefort, X. Tarrago, Phys. Rev. C9, 1018 (1974)

4. D. Glas, U. Mosel, Phys. Rev. C10, 2620 (1974)

5. T. Matsuse, A. Arima, S.M. Lee, Phys. Rev. C26, 2338 ((1982)

6. J.P. Wieleczko, S. Harar, M. Conjeaud, F. Saint-Laurent, Phys. Lett. 93B, 35 (1980)

7. S.M. Lee, T. Matsuse, A. Arime, Phys. Rev. Lett. 45, 165 (1980)

8. R. Vandenbosch, Phys. Lett. 87B, 183 (1979)

9. R. Vandenbosch, A.J. Lazzarini, Phys. Rev. C23, 1074 (1981)

10. O. Civitarese, B.V. Carlson, M.S. Hussein, A. Szanto de Toledo, Phys. Lett. 125B, 22 (1983)

11. J. Gomez del Campo, J.A. Biggerstaff, R.A. Dayras, D. Shapira, A.H. Snell, P.H. Stelson, R.G. Stokstad, Phys. Rev. C29, 1722 (1984)

12. J.D. Hinnefeld, J.J. Kolata, D.J. Henderson, R.V.F. Janssens, D.G. Kovar, K.T. Lesko, G. Rosner, G.S.F. Stephens, A.M. Van den Berg, B.D. Wilkins, F.W. Prosser, S.V. Reinert, P.L. Gonthier, Phys. Rev. C36, 989 (1987)

13. T. Ericson, Adv. Phys. 9, 425 (1960)

14. K.W. Zimmer, Progress Rep. Dept. Heavy Ion Phys. 1986-1987, ed. G. Semenescu, pg. 125-127, I.P.N.E. Bucharest, 1988, unpublished

15. K.W. Zimmer, Ph. D. thesis, I.P.N.E. Bucharest, 1987, unpublished

16. M.N. Namboodiri, E.T. Chulick, J.B. Natowitz, Nucl. Phys. A263, 491 (1976)

17. J. Gomez del Campo, R.G. Stokstad, R.G. Biggerstaff, R.A. Dayras, A.H. Snell, P.H. Stelson, Phys. Rev. C19, 2170 (1979)

18. G. Doukellis, Phys. Rev. C37, 2233 (1988)

19. S. Cohen, F. Plasil, W.J. Swiatecki, Ann. Phys. (N.Y.) 82, 557 (1974)

20. A.J. Sierk, Phys. Rev. C33, 2039 (1986) 
21. H.A. Weidenmüller, Phys. Lett. 10, 331 (1964)

22. M. Ohta, K. Hatogi, S. Okai, Y. Abe, Phys. Rev. C29, 1248 (1984)

23. D.R. Dean, U. Mosel, Z. Phys. A322, 647 (1985)

24. S. Shlomo, J.B. Natowitz, Phys. Lett. 252B, 187 (1990)

25. K.W. Zimmer, Progr. Rep. Dept. Heavy Ion Phys. 1990-1991, eds. C. Ciortea, C. Coca, D. Dumitriu, C. Grama, M. Ionescu-Bujor, M. Pârlog, D. Poenaru, A. Pop, K.W. Zimmer, pg. 46-46, I.P.N.E. Bucharest, 1992, unpublished

26. G. Nebbia, H. Hagel, D. Fabris, Z. Majka, J.B. Natowitz, R.P. Smitt, B. Sterling, G. Mouchaty, G. Berkowitz, K. Strozewski, G. Viesti, P.L. Gonthier, B. Wilkins, M.N. Namboodiri, H. Ho, Phys. Lett. 176B, 20 (1986)

27. D. Fabris, K. Hagel, J.B. Natowitz, G. Nebbia, R. Wada, R. Billerey, B. Cheynis, A. Demeyer, D. Drain, D. Guinet, C. Pastor. J. Alarja, A. Gioni, D. Heuer, C. Morand, B. Viano, C. Mazur, C. Ngo, S. Leray, R. Lucas, M. Rimbag, E. Tomasi, Phys. Lett 196B, 429 (1987)

28. M. Gonin, L. Cooke, K. Hagel, Y. Lou, J.B. Natowitz, R.P. Schmitt, B. Srivastrava, W. Turmel, H. Utsunomiya, R. Wada, B. Fornal, G. Nardelli, G. Nebbia, G. Viesti, R. Zanon, G. Prete, P. Gonthier, B. Wilkins, Phys. Lett. 217B, 406 (1989)

29. C. Gregoire, T.T. S. Kuo, D.B. Stout, Nucl. Phys. A350, 94 (1991)

30. P. Sperr, T.H. Braid, Y. Eisen, D.G. Kovar, F.W. Prosser, J.P. Schiffer, S.L. Tabor, S.E. Vigdor, Phys. Rev. Lett. 37, 321 (1976)

31. M. Conjeaud, S. Garry, S. Harar, J.P. Wieleczko, Nucl. Phys. A309, 515 (1978)

32. I. Tserruya, Y. Eisen, D. Plelte, A. Gavron, H. Öschler, D. Berndt, H.L. Harney, Phys. Rev. C18, 1688 (1978)

33. J.J. Kolata, R.M. Freeman, F. Haas, B. Heusch, A. Gallmann, Phys. Rev. C19, 2237 (1979)

34. D.G. Kovar, D.F. Geesaman, T.H. Braid, Y. Eisen, W. Henning, T.R. Ophel, M. Paul, K.E. Rehm, S.J. Sanders, P. Sperr, J.P. Schiffer, S.L. Tabor, S. Vigdor, B. Zeidman, Phys. Rev. C20, 1305 (1979) 
35. P. Sperr, S. Vigdor, Y. Eisen, W. Henning, D.G. Kovar, T.R. Ophel, B. Zeidman, Phys. Rev. Lett. 36, 405 (1976)

36. N. Poffe, N. Rowley, R. Lindsay, Nucl. Phys. A410, 498 (1983)

37. A. Kabir, M.W. Kermude, N. Rowley, Nucl. Phys. A.481, 94 (1988)

38. C. Jacquemin, S.K. Kataria, Z. Phys. A324, 261 (1986)

39. T. Ugadawa, B.T. Kim, T. Tamura, Phys. Rev. C32, 124 (1985)

40. B.T. Kim, H.C. Kim, K.E. Park, Phys. Rev. C37, 998 (1988)

41. A.K. Mohatny, S.V.S. Sastry, S.K. Kataria, S. Kailas, V.S. Ramamurthy, Phys. Lett. 247B, 215 (1990)

42. M.S. Hussein, Phys. Rev. C30, 1962 (1984)

43. V.L.M. Franzin, M.S. Hussein, Phys. Rev. C38, 2167 (1988)

44. M.A. Nagarajan, G.R. Satchler, Phys. Lett. 173B, 29 (1986)

45. G.R. Satchler, M.A. Nagarajan, J.S. Lilley, I.J. Thompson, Ann. Phys. (N.Y.) 178, $110(1987)$

46. N.C. Francis, K.W. Watson, Am. J. Phys. 21, 659 (1953)

47. H. Feshbach, Ann. Phys. (N.Y.) 5, 357 (1958)

48. H. Feshbach, Ann. Phys. (N.Y.) 19, 357 (1962)

49. H. Feshbach, Ann. Phys. (N.Y.) 43, 357 (1967)

50. M. Tohyama, Phys. Rev. C36, 187 (1987)

51. D. Shapira, R.G. Stokstad, D.A. Bromley, Phys. Rev. C10, 897 (1974)

52. W. Reilly, R. Wieland, A. Gobbi, M.W. Sachs, D.A. Bromley, Nuovo Cim. A 13, 897 (1973)

53. R. Singh, K.A. Eberhard, R.G. Stokstad, Phys. Rev. C22, 1971 (1980)

54. J.V. Maher, M.W. Sachs, R.H. Siemssen, A. Weidinger, D.A. Bromley, Phys. Rev. C188, 1665 (1969)

55. R.G. Stokstad, R.A. Dayras, J. Gomez del Campo, P.H. Stelson, C. Olmer, M.S. Zisman, Phys. Lett. 70B, 289 (1977)

56. F. Saint-Laurent, M. Conjeaud, S. Harar, J.M. Loiseaux, J. Menet, J.B. Viano, Nucl. Phys. A327, 517 (1979)

57. H. Horiuchi, A. Ohnishi. T. Maruyama, Proc. 6th Int. Conf. Nucl. Reaction Mech., Varenna, June 1991, ed. E. Gadioli, pg. 238-247, 
Ricerca Scient. Ed. Permanente Suppl. 84, Milano 1991

58. M. Beckermann, Nucl. Phys. A278, 333 (1977)

59. B. Fornal, F. Gramegna, G. Prete, R. Burch, G.D. D’Erasmo, E.M. Fiore, L. Fiore, A. Pantaleo, V. Paticchio, G. Viesti, P. Blasi, M. Cinausero, F. Lucarelli, M. Anghinolfi, P. Corvisiero, M. Taiuti, A. Zucciati, P.F. Bortignon, D. Fabris, G. Nebbia, J.A. Ruiz, Phys. Rev. C44, 2588 (1991)

60. P. Bonche, S. Levit, D. Vautherin, Nucl. Phys. A427, 278 (1984)

61. P. Bonche, S. Levit, D. Vautherin, Nucl. Phys. A436, 265 (1985)

62. K.W. Zimmer, A. Calboreanu, Rev. Roum. Phys. 32, 521 (1987)

63. K.W. Zimmer, A. Calboreanu, Progr. Rep. Dept. Heavy Ion Phys. 19861987, ed. G. Semenescu, pg. 122-123, I.P.N.E. Bucharest, 1988 unpublished

64. M.G. Mustafa, M. Blann, A.V. Ignatiuk, S.M. Grimes, Phys. Rev. C45, 1078 (1992)

65. K.W. Zimmer, Progr. Rep. Dept. Heavy Ion Phys. 1988-1989, eds. M. Ionescu-Bujor, C. Ciorta, C. Coca, M. Pârlog, K.W. Zimmer, pg. 47-49, I.P.N.E. Bucharest, 1990, unpublished

66. R.W. Hasse, P. Schuck, Nucl. Phys. A445, 2055 (1985)

67. M. Prakash, J. Wambach, Z.Y. Ma, Phys. Lett. 128B, 141 (1983)

68. C. Mahaux, R. Sartor,, Nucl. Phys. A468, 193 (1987)

69. C. Mahaux, R. Sartor,, Nucl. Phys. A475, 247 (1987)

70. C.H. Johnson, C. Mahaux, Phys. Rev. C38, 2589 (1987)

71. R.W. Hasse, P. Schuck, Phys. Lett. 179B, 313 (1986)

72. P.F. Bortignon, C.H. Dasso, Phys. Lett. 189B, 381 (1987)

73. S. Shlomo, J.B. Natowitz, Phys. Rev. C44, 2878 (1991)

74. H.A. Weidenmüller, Nucl. Phys. A471, 1c (1987)

75. L.M. Landau, L.D. Lifchitz, Physique Statisique, pg. 414, Moscow: Nauke 1967

76. W. Dilg, W. Schantl, H. Vonach, M. Uhl, Nucl. Phys. A217, 269 (1973)

77. A.B. Ignatiuk, I.B. Sokolov, I.N. Shubin, Jader. Phys. 5, 989 (1973)

78. N. Vinh Mau, D. Vautherin, Nucl. Phys. A445, 245 (1985) 
79. N. Carlin Filho, M.M. Coimbra, J.C. Acquadro, R. Lignori Neto, E.M. Szanto, E. Farelly-Ressoa, A. Szanto de Toledo, Phys. Rev. C31, 152 (1985)

80. M.K. Grossjean, H. Feldmeier, Nucl. Phys. A444, 113 (1985)

\section{Editorial Remark}

Through a tragic accident the author of this work, 1991/92 guest scientist in KfK and collaborator with our insitute, passed away in March, 16, 1994. The edition of his final work, prepared for publication, follows the original draft and the left suggestions of changements. The final version is prepared by H. Rebel, with helpful advices of H. H. Wolter. 


\section{IN MEMORIAM KARL-WILHELM ZIMMER}

In 16th of March 1994 Karl-Wilhelm Zimmer passed away through a tragic accident whose background features withdraw from simple explanation and arguing. The message of his death in Cologne has petrified us all, his friends and colleagues in Romania, Germany and abroad. It appeared just when we hoped that he will soon integrate in Germany and is going to find a new field for his scientific talent.

Karl-Wilhelm Zimmer was born in October 13, 1951, in Agnita (near Sibiu), Romania. He studied physics at the University of Bucharest and completed his diploma, and then his Ph. D. with a doctoral thesis on the subject: "State and Level Densities of Highly Excited Nuclei" in 1987 in the Institute of Atomic Physics of Bucharest under the scientific guidance of Marin Petrascu. From 1975-1993 he has been appointed scientist in this institute, finally as scientific researcher in the Department of Heavy Ion Physics. Since 1990 he has been the leading scientist of a research group performing experimental spectroscopy of high-energy cosmic ray muons. This research was related to an international collaboration research project (KASCADE in Kernforschungszentrum Karlsruhe), which is focused to observation of cosmic ray induced extensive air showers. In May 1993 he left the Institute of Atomic Physics of Bucharest in view of emigration with his family to Germany.

His scientific interests span the horizon of atomic and nuclear physics problems, specifically with an improved understanding of formation and decay of the compound nucleus and the level density of highly excited nuclei. In addition he developed dedicated particle detection techniques and contributed to the technical achievements of Bucharest tandem and postacceleration system. In 1987 he received the Dragomir-Hurmuzescu prize of the Romanian Academy of Sciences. Finally, distinctly changing the research field, in a situation and period when most among us would have had avoided any personal risk, he took the responsibility in establishing an astrophysically motivated research project, exploring experimentally the spectroscopic response of an electromagnetic calorimeter to high-energy cosmic ray muons. He did so since he had obviously recognized the chance to extend the research possibilities of the institute to nonaccelerator particle physics, being embedded in a larger international collaboration. His courage following this view deserves our respect and admiration. 
When he decided to emigrate to Germany where he had many relatives living, this decision has not been easy for him. He discussed the arguments and the implications for the research project in a fair way with his friends and collaborators. Born in Romania, the country which he loved as his home of childhood, education and studies and where he married and his son was born, and being from German origin he felt himself as a mediator, pointing to the common roots of our culture, cultivating mutual respect and understanding of different mentalities. He could suffer very much from realizing small-minded thinking and discriminating behaviour, distorting a common responsibility for the reality to accuses of the neighbours. It might have been the tragedy of his life that his modesty was too reluctant to express his personal suffers to helping friends.

In Karl-Wilhelm Zimmer we have lost a highly respected scientist, a kind colleague and a dear friend.

\section{Rubl}

(H. Rebel) 


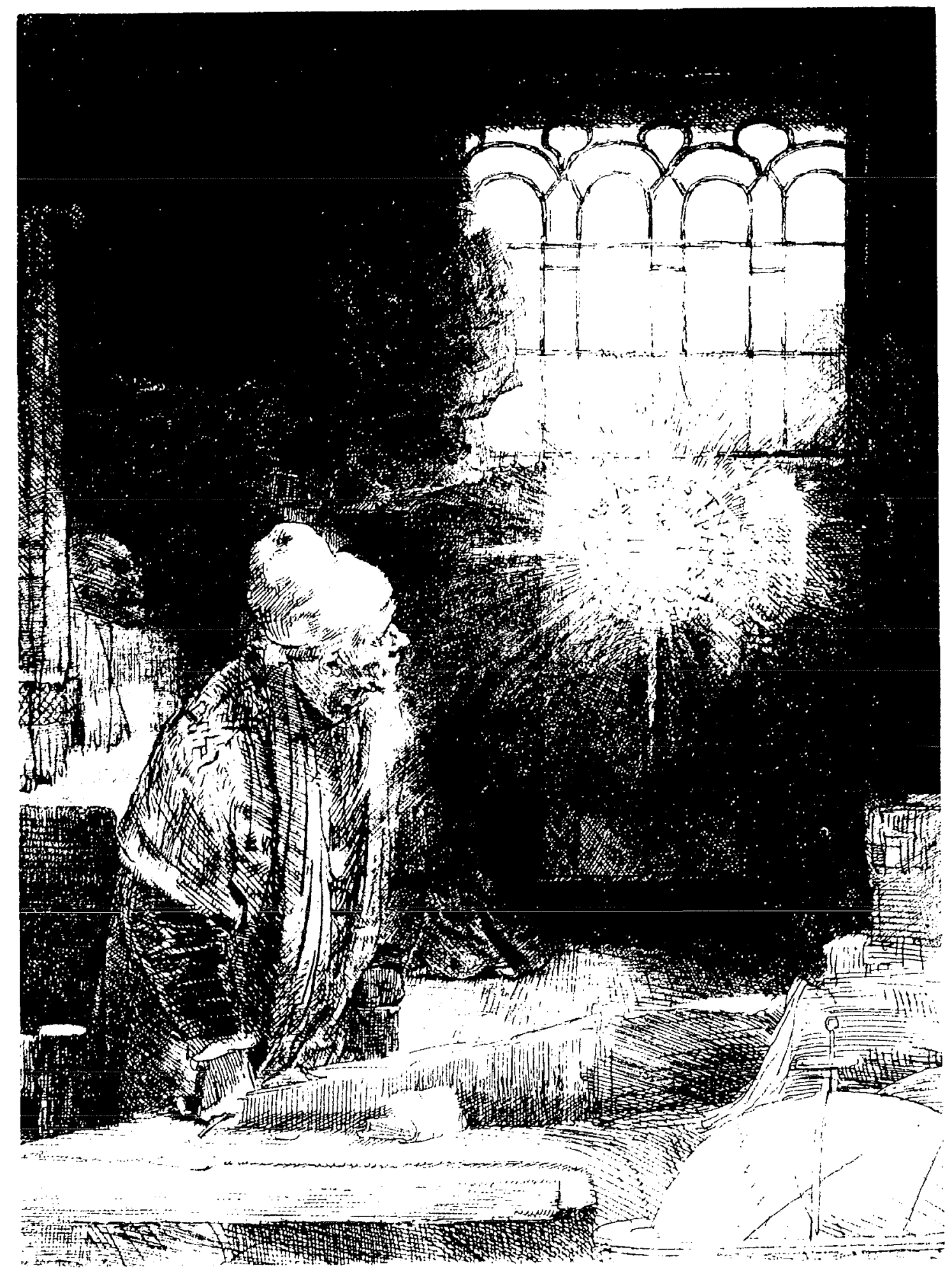

